\begin{tabular}{|l|l|}
\hline $\begin{array}{l}\text { 2. To: (Receiving Organization) } \\
\text { Distribution }\end{array}$ & $\begin{array}{l}\text { 3. From: (Originating Organization) } \\
\text { BL Philipp Cogema Engineering Corp }\end{array}$ \\
\hline $\begin{array}{l}\text { 5. Proj./Prog./Dept./Div.: } \\
\text { Spent Nuclear Fuels Project }\end{array}$ & $\begin{array}{l}\text { 6. Design Authorlty/Design Agent/Cog. Engr.: } \\
\text { R Whitehurst }\end{array}$ \\
\hline
\end{tabular}

8. Originator Remarks:

For document approval and release

Design verfication methods for OCRLM compments are by independent revicu in accordance with EN 6- $6091 \% \phi 2$. Documentation of this nevew is accomplisted by the signatures heldw anthis EDT.
11. Recelver Remarks:
11A. Design Baseline Document? $\bigcirc$ Yes
O No

\begin{abstract}
4. Related EDT No.:
NA

7. Purchase Order No.:

NA

9. Equip./Component No.:

CVD
\end{abstract}

10. System/BIdg./Facility:

CVDF/ $142 \mathrm{~K}$

12. Major Assm. Dwg. No.:

NA

13. Permit/Permit Application No.:

NA

14. Required Response Date:

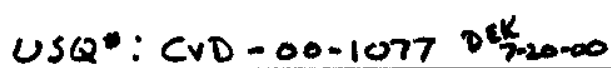
$6 / 15 / 00$

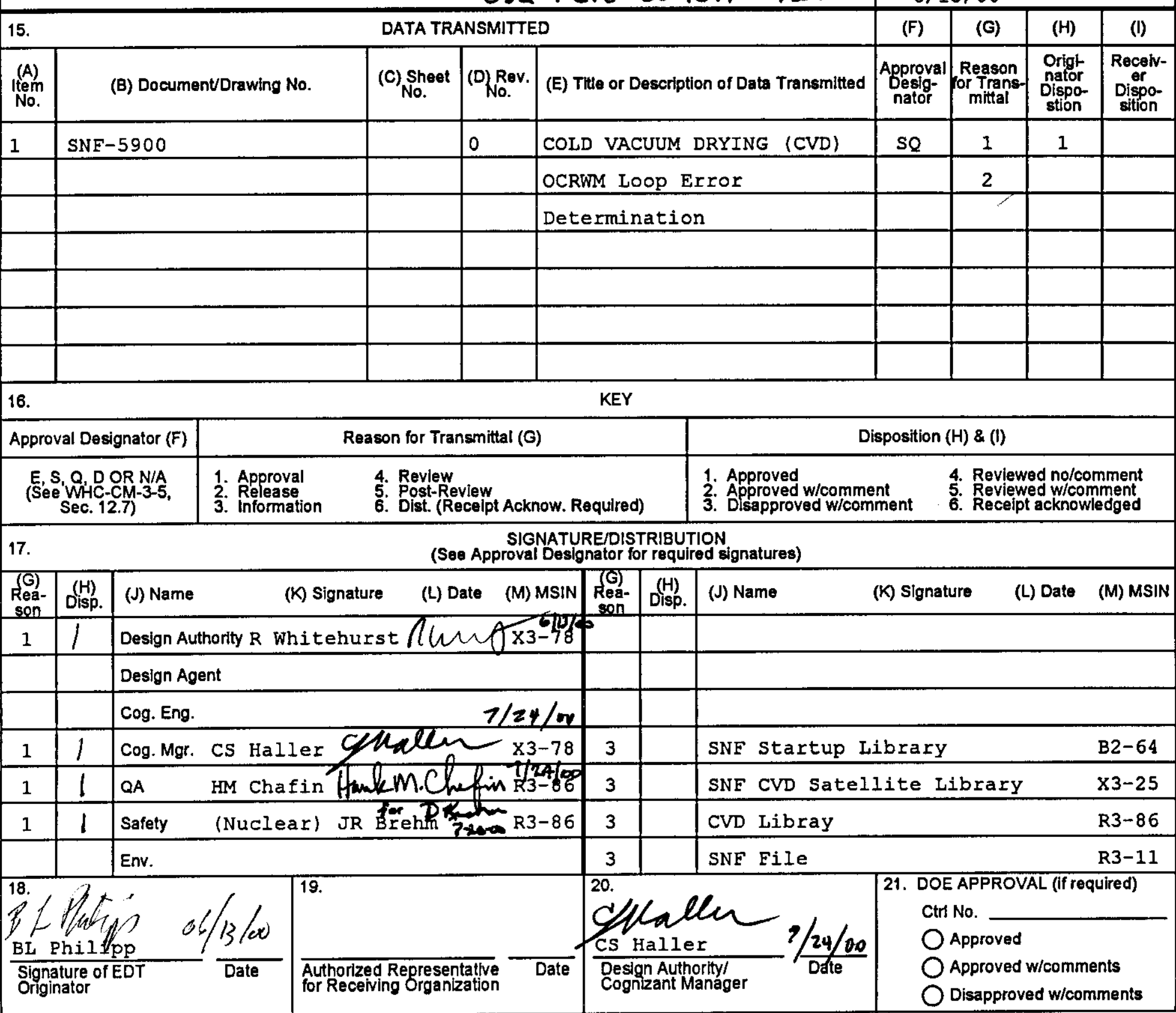


SNF-5900

Revision 0

\section{Cold Vacuum Drying \\ (CVD) OCRWM Loop Error Determination}

Prepared for the U.S. Department of Energy

Assistant Secretary for Environmental Management

Project Hanford Management Contractor for the

U.S. Department of Energy under Contract DE-AC06-96RL13200

Fuor Hanford

P.O. Box 1000

Richland, Washington 
SNF-5900

Revision 0

EDT 624970

\section{Cold Vacuum Drying (CVD) OCRWM Loop Error Determination}

Project No: W441

Document Type: TI

Division: SNF

BL Philipp

Fluor Hanford

Date Published

June 2000

Prepared for the U.S. Department of Energy

Assistant Secretary for Environmental Management

Project Hanford Management Contractor for the

U.S. Department of Energy under Contract DE-AC06-96RL13200

Fluor Hanford

P.O. Box 1000

Richland, Washington

$\frac{\text { Hncofolden }}{\text { Release Approval }} \frac{7 / 26100}{\text { Date }}$

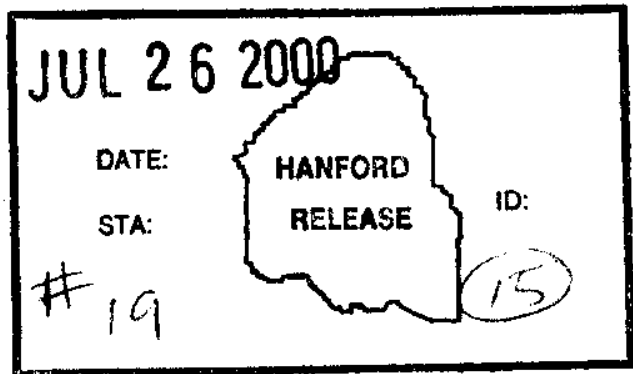

Release Stamp 
TRADEMARK DISCLAIMER

Reference herein to any specific commercial product, process, or service by trade name, trademark, manufacturer, or otherwise, does not necessarily constitute or imply its endorsement, recommendation, or favoring by the United States Government or any agency thereof or its contractors or subcontractors.

This report has been reproduced from the best available copy.

Total Pages: $\quad 34$ 


\section{Cold Vacuum Drying (CVD)}

\section{OCRWM Loop Error Determination}

Project W-441

SNF-5900

June 2000

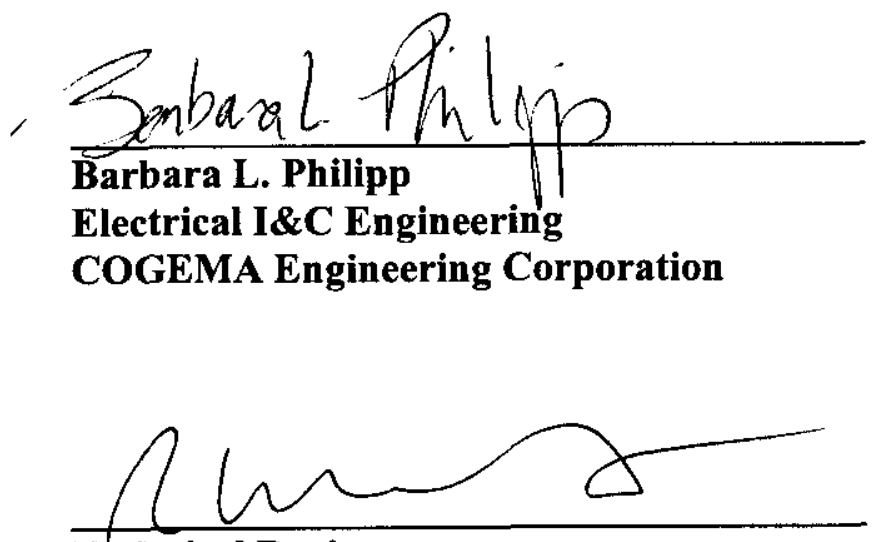

Technical Reviewer

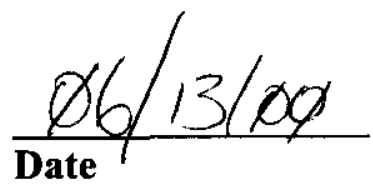

Richard Whitehurst II

Technical Integration

Fluor Hanford, Incorporated 


\section{REVIEW CHECKLIST}

Document Reviewed:

SNE-5900 Rev. 0, Cold Vacuum Drying (CVD) OCRWM Loop Error Determination Project W-441

Scope of Review:

Independant, Engineering review of all calculations.

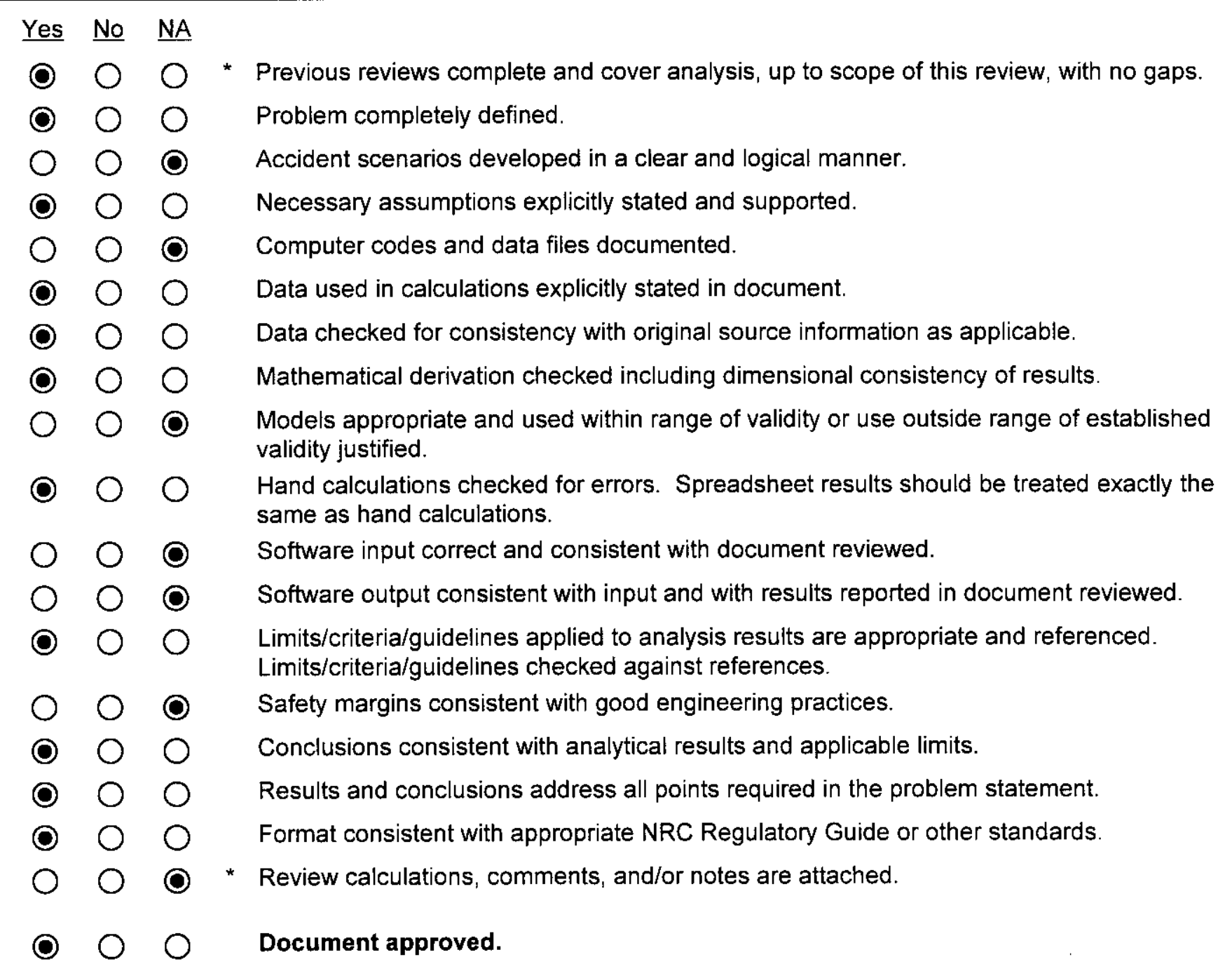

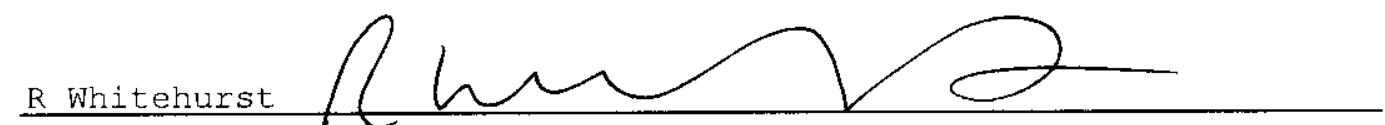

Reviewer (Printed Name and Signature)

$\frac{7 / 19 / 00}{\text { Date }}$

*Any calculations, comments, or notes generated as part of this review should be signed, dated and attached to this checklist. Such material should be labeled and recorded in such a manner as to be intelligible to a technically qualified third party. 


\section{Contents}

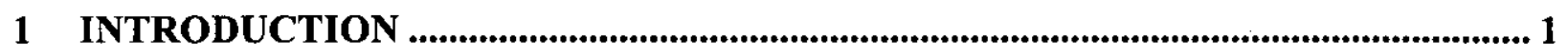

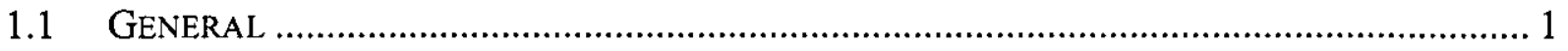

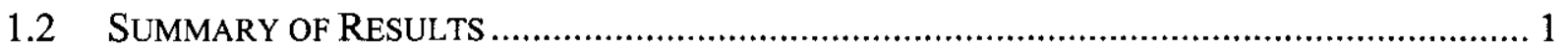

2 MCO PRESSURE TRANSMITTER TO MCS ........................................................... 3

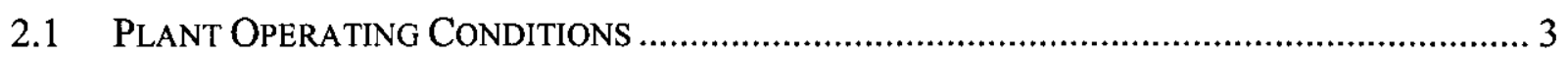

2.1.1 Proof Mode and Final Pressure Test ……….................................................... 3

2.2 ABSOlUte PreSSURE TRANSMITTER (PT-1 *08, PT-1*10) ……..................................... 3

2.2.1 Instrument Data............................................................................................ 4

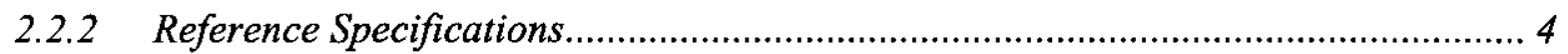

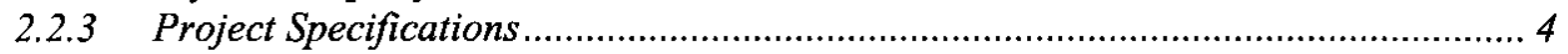

2.2.4 Uncertainty Terms that Affect Set Point Determination.......................................... 4

2.3 FRAMATOME STAR SYSTEM - ANALOG Voltage Isolation Module (AVIM) …......... 8

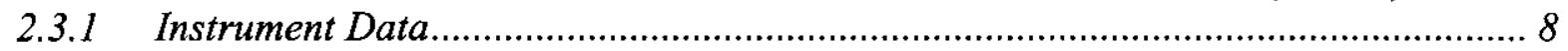

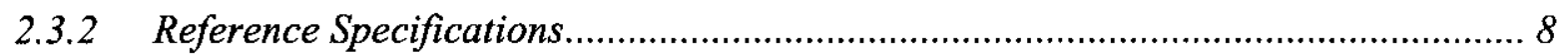

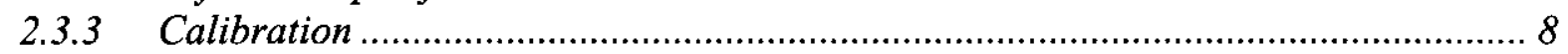

2.4 MCS ANALOG TO DigiTAL Module (A/D) ........................................................... 10

2.4.1 Instrument Data

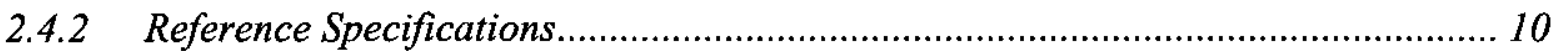

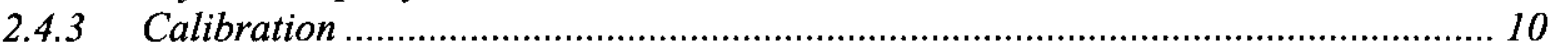

2.5 TOTAL LOOP UNCERTAINTY - GENERAL SERVICE MCS LOOP ...................................... 12

2.6 MCS VACUUM LOOP ERROR DETERMINATION (OCRWM)........................................... 12

2.7 DELTA PRESSURE ACCURACY ............................................................................. 13

2.7.1 Uncertainty Terms that Affect Accuracy ........................................................ 13

2.7.2 MCS Delta Vacuum Loop Error Determination (OCRWM) ................................. 14

2.8 OCRWM VACUUM PRESSURE CALIBRATION REQUIREMENTS ........................................ 15

2.8.1 Vacuum Pressure Sensor/Loop ..................................................................... 15

2.8.2 Measurement and Test Equipment …………..................................................... 17

3 MCO TEMPERATURE TRANSMITTER TO MCS ....................................................... 18

3.1 PLANT OPERATING CONDITIONS ......................................................................... 18

3.1.1 Drying Mode Operations............................................................................... 18

3.2 TEMPERATURE TRANSMITTER (TIT-3*05, TIT-3*12) ................................................ 19

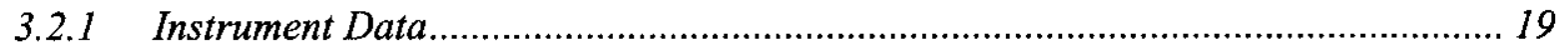

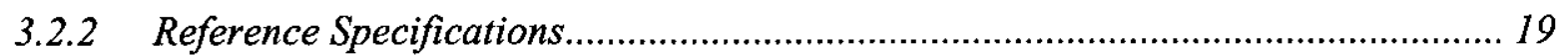

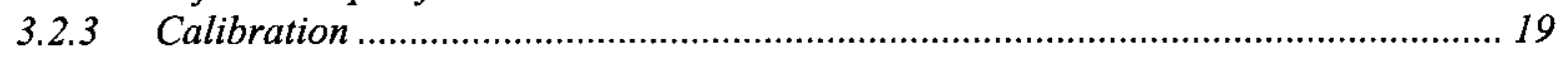

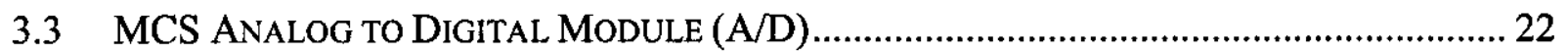

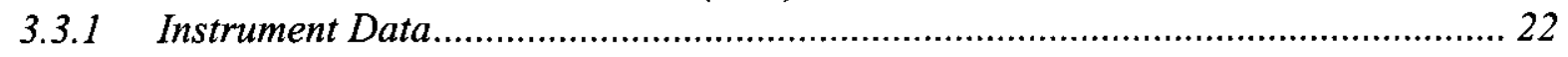

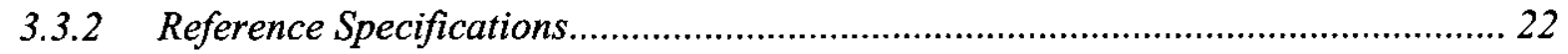

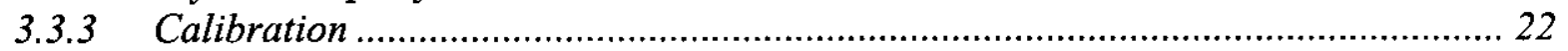


3.4 TOTAL LOOP UNCERTAINTY - GENERAL SERVICE MCS LOOP ...................................... 23

3.5 MCS TW TEMPERATURE LOOP ERROR DETERMINATION (OCRWM) ............................ 23

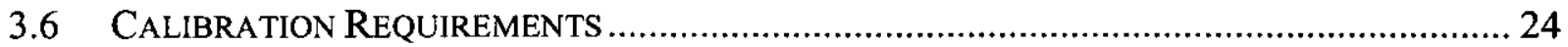

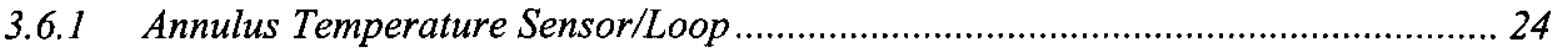

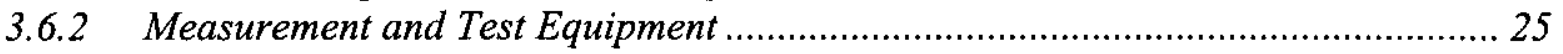

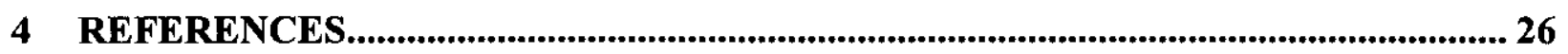

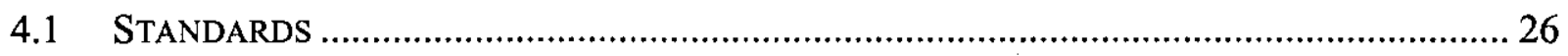

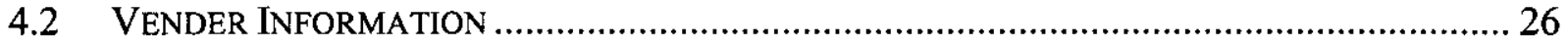

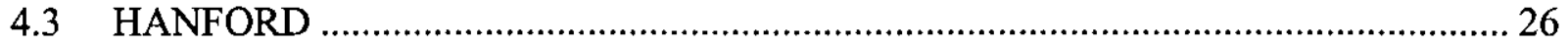

\section{List of Tables}

Table 1-1. OCRWM Loop Error ............................................................................................. 1

Table 1-2. Calibration Requirements .................................................................................. 2

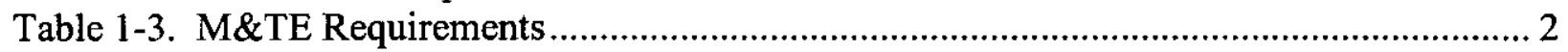

\section{List of Figures}

Figure 2.1 Baratron Absolute Pressure Simplified Block Diagram .......................................... 3

Figure 3.1 TW Temperature Simplified Block Diagram.............................................................. 18 


\section{List of Acronyms and Abbreviations}

$\begin{array}{ll}\text { AL } & \text { Analytical Limit } \\ \text { AU } & \text { Accuracy Uncertainty } \\ \text { B } & \text { Bias } \\ \text { CS } & \text { Calibrated Span } \\ \text { CU } & \text { Channel Uncertainty } \\ \text { DAC } & \text { Data Acquisition } \\ \text { DR } & \text { Drift Effect } \\ \text { HE } & \text { Humidity Effect } \\ \text { FS } & \text { Full Scale } \\ \text { MCS } & \text { Monitoring and Control System } \\ \text { mR } & \text { milli-Rad } \\ \text { LT } & \text { Loop Tolerance } \\ \text { MTE } & \text { Measurement and Test Equipment } \\ \text { NPH } & \text { National Phenomena Hazard } \\ \text { PC } & \text { Performance Category: } \\ \text { Psig } & \text { Pounds per Square Inch - Gauge } \\ \text { PSE } & \text { Power Supply Effect } \\ \text { RA } & \text { Relative Accuracy } \\ \text { RD } & \text { Reading Error } \\ \text { Rdg } & \text { Reading } \\ \text { RE } & \text { Radiation Effect } \\ \text { RH } & \text { Relative Humidity } \\ \text { SA } & \text { Setting Accuracy } \\ \text { SC } & \text { Safety Class } \\ \text { SCC } & \text { Standard cubic centimeters } \\ \text { SCIC } & \text { Safety Class Instrumentation and Controls } \\ \text { SE } & \text { Seismic Effect } \\ \text { SMTE } & \text { Sensor Measurement and Test Equipment Effect } \\ \text { SP } & \text { Static Pressure Effect } \\ \text { TDR } & \text { Turn Down Ratio } \\ \text { TE } & \text { Temperature Effect } \\ \text { TEM } & \text { Test Equipment Error Maximum } \\ \text { TID } & \text { Total Integrated Dose } \\ \text { URL } & \text { Upper Range Limit } \\ \text { VDC } & \text { Volts - Direct Current } \\ & \end{array}$




\section{Introduction}

\subsection{General}

Characterization is specifically identified by the Richland Operations Office (RL) for the Office of Civilian Radioactive Waste Management (OCRWM) of the U.S. Department of Energy (DOE), as requiring application of the requirements in the Quality Assurance Requirements and Description (QARD) (RW-0333P DOE 1997a). Those analyses that provide information that is necessary for repository acceptance require application of the QARD.

The CVD project identified the loops that measure, display, and record MCO vacuum pressure and Tempered Water (TW) temperature data as providing OCRWM data per Application of the Office of Civilian Radioactive Waste Management (OCRWM) Quality Assurance Requirements to the Hanford Spent Nuclear Fuel Project HNF-SD-SNF-RPT-007. Vacuum pressure transmitters (PT $1 * 08,1 * 10$ ) and TW temperature transmitters (TIT- $3 * 05,3 * 12$ ) are used to verify drying and to determine the water content within the multi-canister overpack $(\mathrm{MCO})$ after cold vacuum drying (CVD).

\subsection{Summary of Results}

Table 1-1 below summarizes the OCRWM data loop crrors for the Pressure Test and the Proof Demonstration. For both of these assessments, the MCO annulus water temperature must be $\geq 40^{\circ} \mathrm{C}$ as measured by TIT $-3 * 05,3 * 12$, and the MCO pressure must be $\leq 12$ torr. Additionally for the Final Rebound Pressure Rise Test, the pressure must be maintained $\leq 12$ torr, and the MCO pressure rise must be $\leq 2.4$ torr $/ \mathrm{hr}$ for $\geq 1$ hour.

Table 1-1. OCRWM Loop Error

\begin{tabular}{|l|c|c|c|}
\hline \multicolumn{1}{|c|}{$\begin{array}{c}\text { OCRWM } \\
\text { Evaluation }\end{array}$} & $\begin{array}{c}\text { OCRWM } \\
\text { Data to MCS }\end{array}$ & Field sensor & $\begin{array}{c}\text { Total Expected } \\
\text { reading error (during } \\
\text { calibration period) }\end{array}$ \\
\hline Proof Demonstration & MCO Vacuum pressure & PT $1 * 08,1^{*} 10$ & $\begin{array}{c} \pm 1.98 \text { torr with } 2 \text { torr } \\
\text { system drop }\end{array}$ \\
\hline $\begin{array}{l}\text { Initial } 1 / \text { Final Rebound } \\
\text { Pressure Test }\end{array}$ & $\begin{array}{c}\text { MCO Delta Vacuum } \\
\text { pressurc }\end{array}$ & PT $1^{*} 08,1^{*} 10$ & \pm 0.23 torr \\
\hline $\begin{array}{l}\text { Proof Demonstration } \\
\& \text { Pressure Test }\end{array}$ & TW temperature & TIT- $3^{*} 05,3^{*} 12$ & $\pm 0.58^{\circ} \mathrm{C}$ \\
\hline
\end{tabular}

\footnotetext{
${ }^{1}$ Not OCRWM data. The Initial Rebound Pressure Test is safety related information used in processing.
} 
Table 1-2 below summarizes the sensor tolerances required during calibration to support the OCRWM loop error calculations.

Table 1-2. Calibration Requirements

\begin{tabular}{|c|c|c|c|}
\hline Field sensor & Ranges & Sensor Tolerance & $\begin{array}{c}\text { Loop Tolerance } \\
\text { (OCRWM) }\end{array}$ \\
\hline PT $1^{*} 08,1^{*} 10$ & $\leq 10$ Torr & \pm 0.02 torr & \pm 0.1 torr \\
\cline { 2 - 4 } & 10 to 100 Torr & \pm 0.15 torr & \pm 0.2 torr \\
\hline TIT $-3^{*} 05,3^{*} 12$ & 0 to $100^{\circ} \mathrm{C}$ & $\pm 0.17^{\circ} \mathrm{C}$ & $\pm 0.2^{\circ} \mathrm{C}$ \\
\hline
\end{tabular}

Table 1-3 below summarizes the Measurement and Test Equipment (M\&TE) accuracies required during calibration to support the OCRWM loop error calculations. Total crror (square root sum of squares) for the actual equipment used shall be less than or equal to the assumed instruments. Failure to meet these requirements may impact the total expected loop errors within the calibration period. Therefore, failure to meet these requirements may impact the data used for the Proof of Dryness acceptance criteria.

Table 1-3. M\&TE Requirements

\begin{tabular}{|c|c|c|c|c|}
\hline $\begin{array}{c}\text { Test } \\
\text { Equipment }\end{array}$ & \multicolumn{2}{|c|}{ Relative Accuracy } & $\begin{array}{c}\text { Reading } \\
\text { Error }\end{array}$ & $\begin{array}{c}\text { Test Equipment } \\
\text { Error Maximum } \\
\text { (TEM) }\end{array}$ \\
\cline { 2 - 5 } & $\begin{array}{c}\text { M\&TE } \\
\left(\mathrm{RA}_{\mathrm{MTE}}\right)\end{array}$ & $\begin{array}{c}\text { Calibration } \\
\text { Standard (RA }\end{array}$ & $\begin{array}{c}\text { Assume digital } \\
\text { readout }\end{array}$ & $\begin{array}{c}\text { Square Root Sum of } \\
\text { Squares }\end{array}$ \\
\hline $\begin{array}{c}\text { Volt Metcr } \\
\text { (Fluke 743B) }\end{array}$ & $\pm 0.0415 \% \mathrm{CS}$ & $\pm 0.0265 \% \mathrm{CS}$ & $0 \% \mathrm{FS}$ & $\pm 0.05 \% \mathrm{CS}$ \\
\hline $\begin{array}{c}\text { Vacuum Transfer } \\
\text { Standard } \\
\text { (MKS PBTS1) }\end{array}$ & $\pm 0.05 \% \mathrm{CS}$ & $\pm 0.01 \% \mathrm{CS}$ & $0 \% \mathrm{FS}$ & $\pm 0.05 \% \mathrm{CS}$ \\
\hline $\begin{array}{c}\text { Temperature } \\
\text { Transfer Standard } \\
\text { (AMETEK 140SE } \\
\text { JOFRA dry block) }\end{array}$ & $\pm(0.4) \% \mathrm{CS}$ & $\pm 0.1 \% \mathrm{CS}$ & $0 \% \mathrm{FS}$ & $\pm 0.4 \% \mathrm{CS}$ \\
\hline
\end{tabular}

Selection of alternate M\&TE instruments will require

1.) An assessment of the total instrument error contribution (as described in Table 1-3) to assure that the acceptance criteria is not affected or

2.) Development of an updated acceptance criteria. 


\section{MCO pressure transmitter to MCS}

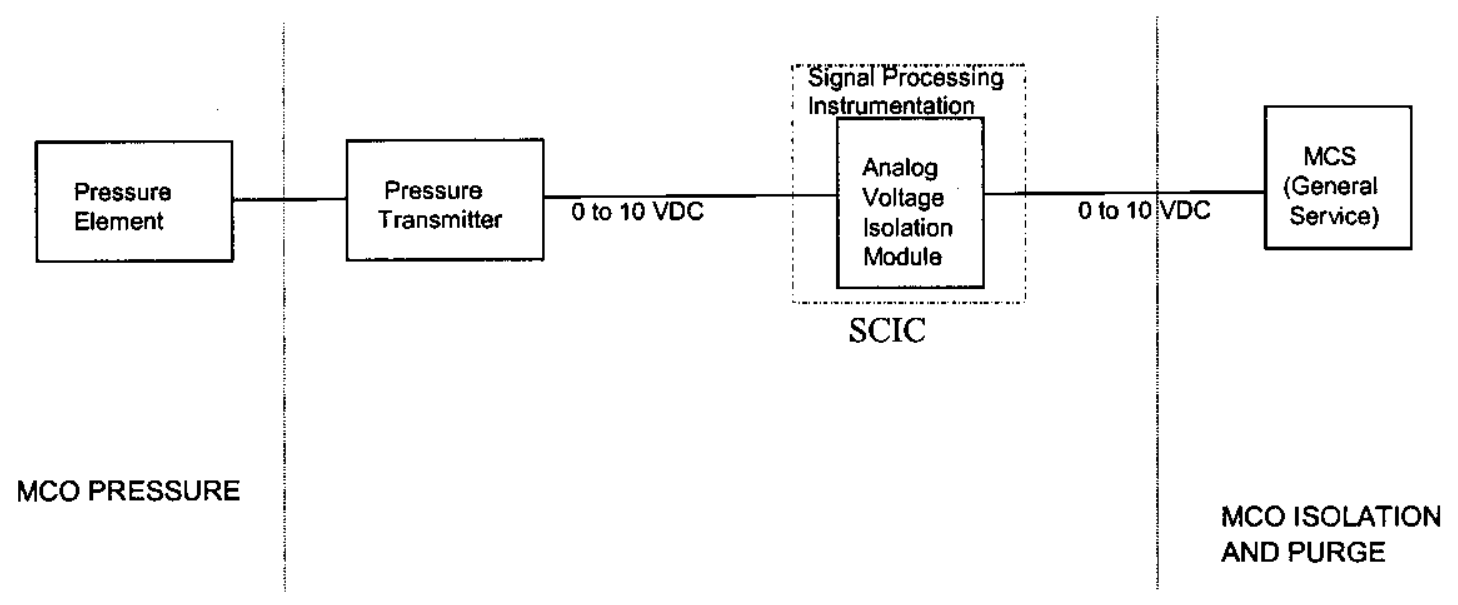

Figure 2.1 Baratron Absolute Pressure Simplified Block Diagram

\subsection{Plant Operating Conditions}

MCO absolute pressure, in combination with the tempered water (TW) water temperature, provides an indication of water content in the MCO. This information is required for the Office of Civilian Radioactive Waste Management (OCRWM).

\subsubsection{Proof Mode and Final Pressure Test}

Once the MCO passes a initial pressure rebound test, an evacuation to less than 12 torr for up to 28 hours (for an MCO with two scrap baskets) is then conducted with the expected system base pressure of $<0.5$ torr. This is called the "PROOF" mode of operation. The proof mode requires the use of the vacuum pump to remove any trapped water in cracks in the fuel. At the completion of the PROOF MODE, a final rebound test is conducted. This post drying operation at $>40^{\circ} \mathrm{C}$ is utilized to verify that $<200 \mathrm{~g}(0.44 \mathrm{lb})$ of water is present in the MCO prior to shipment to the CSB (see HNF-1851, Cold Vacuum Drying Residual Free Water Test Description).

\subsection{Absolute Pressure Transmitter (PT-1*08, PT-1*10)}

The sensor chosen to monitor pressure for the 12 torr analytical limit is a high accuracy; temperature regulated Baratron ${ }^{\mathrm{T} M}$ Absolute Pressure Transmitters from MKS Instruments.

\footnotetext{
${ }^{\mathrm{T}}$ Baratron is a registered trademark of MKS Instruments, Inc, Andover, MA.
} 
Baratron Absolute Pressure Transmitter

\subsubsection{Instrument Data}

Name: Baratron Absolute Pressure Transmitter

Manufacturer: MKS Instruments, Inc.

Model Number: 427A A 00100

Type: High Accuracy, Temperature Controlled $\left(45^{\circ} \mathrm{C}\right)$ Absolute Pressure Transmitter

\subsubsection{Reference Specifications}

Range: 0 to 100 Torr

(Bulletin 400-10/96, 1996 - MKS Instruments, Inc.)

Resolution: $1 \times 10^{-4}$ of Full Scale

Accuracy: $0.15 \%$ of Reading \pm temperature coefficient.

Temperature Effect: Zero $0.007 \% \mathrm{FS} /{ }^{\circ} \mathrm{C}$

$$
\text { Span } 0.020 \% \operatorname{Rdg} /{ }^{\circ} \mathrm{C}
$$

Temperature Limits: 15 to $40^{\circ} \mathrm{C}\left(60\right.$ to $\left.105^{\circ} \mathrm{F}\right)$

Uncertainty below range $15^{\circ} \mathrm{C}\left(60^{\circ} \mathrm{F}\right)= \pm 0.05$ torr

(letter from R. Traverso MKS Applications Engineer 1/26/98)

Power Supply: \pm 15 VDC $\pm 5 \%$

Input/Output: Range: 0.0 to $10.0 \mathrm{VDC}$

Sensor Overpressure limit: 35 psia

Factory Mutual Approvals: Explosion-proof: Class 1 Division 1\&2, Group C \& D

Calibration Interval: 1 year between calibrations

\subsubsection{Project Specifications}

The project safety specifications for the 12 torr sensor include the following:

Safety Classification: Safety Class (SC)

Performance Category: PC-3 (for pressure boundary only)

Environmental Qualification: Environmental Condition B (Certified to $105^{\circ} \mathrm{F}$ )

NPH Design Requirements: Seismic Condition B for boundary only

Required Safety Functions: Pressure boundary integrity, input to SCIC for 12 torr trip and pressure rebound test results

\subsubsection{Uncertainty Terms that Affect Set Point Determination}

A. Assumptions
i.) Turn Down Ratio $=1$ (transmitter will have a full span of 100 Torr)
ii.) Calibrations performed at ambient $72 \pm 8^{\circ} \mathrm{F}$ or 64 to $80^{\circ} \mathrm{F}$
iii.) $\mathrm{Min} / \mathrm{max}$ temperature range is 40 to $115^{\circ} \mathrm{F}$
iv.) Drift: $\pm 1 \%$ Calibrated Span (CS) per year
v.) Calibrated Span (CS) $=100$ Torr 
B. Sensor Uncertainty

i.) Relative Accuracy (RA)

Given: $\mathrm{RA}=0.15 \%$ Reading $(\mathrm{Rdg})$

ii.) Static Pressure (SP)

$\mathrm{SP}=0 \% \mathrm{CS}$

Basis: Assumed zero for pressures $<35$ psi.

Sensor maintains $\mathrm{RA}= \pm 0.15 \%$ of $\mathrm{Rdg}$ up to $35 \mathrm{psi}$

iii.) Humidity Effect (HE)

$\mathrm{HE}=0 \%$ Calibrated Span

Basis: Housed in NEMA 4 enclosure

iv.) Temperature Effect (TE)

Given: $\mathrm{TE}_{\mathrm{s}}= \pm 0.020 \% \mathrm{Rdg} /{ }^{\circ} \mathrm{C}$ for Span (100 Torr)

$\mathrm{TE}_{\mathrm{z}}= \pm 0.007 \% \mathrm{FS} /{ }^{\circ} \mathrm{C}$ for Zero (0 Torr)

Uncertainty below temp range of $15^{\circ} \mathrm{C}\left(60^{\circ} \mathrm{F}\right)= \pm 0.05$ torr

With $\mathrm{TDR}=1, \mathrm{CS}=\operatorname{Span}=100$ Torr

$$
\begin{aligned}
& \text { Assume worst case Rdg =100 } \begin{array}{l}
\mathrm{TE}_{\mathrm{S}}=0.02 \% \mathrm{CS} /{ }^{\circ} \mathrm{C} \\
\mathrm{TE}_{\mathrm{z}}=0.007 \% \mathrm{CS} /{ }^{\circ} \mathrm{C}
\end{array} \\
& \begin{array}{r}
\text { Combining terms (+) } \\
\text { Temp Effect }=0.007 \% * \mathrm{FS} /{ }^{\circ} \mathrm{C}+0.02 \% * \mathrm{Rdg} /{ }^{\circ} \mathrm{C}=0.027 \% \mathrm{CS} /{ }^{\circ} \mathrm{C}
\end{array} \\
& \begin{array}{r}
\text { Temp Uncertainty }=0.027 \% \mathrm{CS} /{ }^{\circ} \mathrm{C}+0.05 * 100 \% / 100 \text { torr } \text { (converts to } \mathrm{CS} \\
\text { since calibrated range is } 100 \text { torr) }
\end{array} \\
& =0.027 \% \mathrm{CS} /{ }^{\circ} \mathrm{C}+0.05 \% \mathrm{CS}
\end{aligned}
$$

Assume $\operatorname{Tmax}=105^{\circ} \mathrm{F}$, Tnorm (lower) $=72-8=64^{\circ} \mathrm{F}$

Therefore Tdelta $=105^{\circ} \mathrm{F}-64^{\circ} \mathrm{F}=41 \Delta^{\circ} \mathrm{F}=22.8 \Delta^{\circ} \mathrm{C}$

i.e., Temp Uncertainty $=(0.027 * \mathrm{FS} * \mathrm{dT}+0.05 * \mathrm{FS}) \%$

$$
\begin{aligned}
& =\left(0.027 * 100^{*} 22.8^{\circ} \mathrm{C}+0.05^{*} 100\right) / 100 \\
& =0.666 \% \mathrm{CS}
\end{aligned}
$$

v.) Radiation Effect (RE)

Given: Dose Rate $=8.5 \mathrm{mR} / \mathrm{hr}$

Total Integrated Dose $=8.5 \mathrm{mR} / \mathrm{hr} * 43800 \mathrm{hr}=372,300 \mathrm{mR}$

No Radiation Effect Data, assumed zero because relatively small dose

Assume: $\mathrm{RE}= \pm 0 \% \mathrm{CS}$

vi.) Power Supply Effect (PSE)

Given: No PSE while supply voltage maintained at $15 \mathrm{VDC} \pm 5 \%$

$\mathrm{PSE}=0 \%$ 
Baratron Absolute Pressure Transmitter

vii.) Seismic Effect (SE)

No credit for this device is taken during or after a seismic event

$\mathrm{SE}=0 \%$

viii.) Sensor Measurement and Test Equipment Effect (SMTE)

Test Equipment1 : Fluke 743B, DC Volts, Damping is OFF

Calibration Temperature Range with no TempEffect: $>18$ and $<28^{\circ} \mathrm{C}$

Full Scale (FS) $=10 \mathrm{~V}$

( $\sim 64$ to $\left.80^{\circ} \mathrm{F}\right)$

Calibrated Span $=0$ to $10 \mathrm{~V}$, Max Reading $(\mathrm{Rdg})=100$ Torr $=10 \mathrm{~V}$

$\mathrm{RA}_{\mathrm{MTE1}}=0.025 \% * \mathrm{Rdg}+3 *(0.005 \% \mathrm{FS})($ Given: times 3 with damping OFF)

$=0.025 \% * \mathrm{CS}+0.015 \% \mathrm{FS} *(\mathrm{CS} / \mathrm{FS})$

$= \pm 0.040 \% \mathrm{CS}$

$\mathrm{RA}_{\mathrm{STD} 1}=0.01 \% * \mathrm{Rdg}+3 * 0.005 \% \mathrm{FS}$ (Use given Source Accuracy,

$=0.01 \% * \mathrm{CS}+0.015 \mathrm{FS} *(\mathrm{CS} / \mathrm{FS})$

Assume also *3 with Damping OFF)

$= \pm 0.025 \% \mathrm{CS}$

$\mathrm{RD} 1=0 \% \mathrm{FS}$ (digital readout)

$\mathrm{SAl}=0.15 \% \mathrm{Rdg}+\mathrm{TE}$ (relative accuracy and TempEffect

Assume calibrated at 72 and operated at 64 to 80 )

$=0.15 \% \mathrm{Rdg}+0.027 \% \mathrm{CS} /{ }^{\circ} \mathrm{C}^{*} \Delta \mathrm{T}=0.15 \% \mathrm{CS}+\left(0.027 \% \mathrm{CS} /{ }^{\circ} \mathrm{C}\right)^{*} 8.89{ }^{\circ} \mathrm{C} / 2$

$=0.27 \% \mathrm{CS}$

$\mathrm{MTE} 1=\left(\mathrm{RA}_{\mathrm{MTE} 1}{ }^{2}+\mathrm{RA}_{\mathrm{STD} 1}{ }^{2}+\mathrm{RD}^{2}+\mathrm{SA}^{2}\right)^{1 / 2}$

MTE1 $=\left(0.04^{2}+0.025^{2}+0^{2}+0.27^{2}\right)^{1 / 2}=0.274 \% \mathrm{CS}$

$\mathrm{MTE} 1=0.274 \% \mathrm{FS}$

Test Equipment2, MKS Portable Pressure Transfer Standard Type PBTS1

Operating Temperature Range: 15 to $40^{\circ} \mathrm{C}$

Full Scale (FS) $=100$ Torr

Resolution $=0.0001$ Torr

$$
\begin{aligned}
\mathrm{RA}_{\mathrm{MTE} 2} & =0.05 \% * \mathrm{Rdg} \\
& =0.05 \% * \mathrm{CS}(100 \text { Torr }=\mathrm{CS} \text { is maximum Reading }) \\
& = \pm 0.05 \% \mathrm{CS}
\end{aligned}
$$

RAsTD2 $<=0.01 \%$ CS (Assume Calibration Standard used was at least 4:1)

$\mathrm{RD} 2=0 \% \mathrm{FS}$ (digital readout)

$\mathrm{SA} 2=0$ (already counted in MTE1)

MTE2 $=\left(\mathrm{RA}_{\mathrm{MTE}}{ }^{2}+\mathrm{RA}_{\mathrm{STD1}}{ }^{2}+\mathrm{RD}^{2}+\mathrm{SAl}^{2}\right)^{1 / 2}$

MTE2 $=\left(0.05^{2}+0.01^{2}+0^{2}+0^{2}\right)^{1 / 2}=0.051 \% \mathrm{CS}$

$\mathrm{MTE} 2=0.051 \% \mathrm{FS}$

SMTE $=$ MTE1 + MTE2 $=0.274+0.051=0.325 \% \mathrm{CS}$ 
Baratron Absolute Pressure Transmitter

ix.) Total Sensor Uncertainties

The assumed drift term DR will be taken as a bias (conservative option).

$$
\begin{aligned}
\mathrm{e}^{+} & =\left[(\mathrm{RA}+\mathrm{TE})^{2}+\mathrm{SP}^{2}+\mathrm{HE}^{2}+\mathrm{RE}^{2}+\mathrm{PSE}^{2}+\mathrm{SE}^{2}+\mathrm{SMTE}^{2}\right]^{1 / 2}+\mathrm{DR} \\
& =\left[(0.15+0.666)^{2}+0+0+0+0+0+(0.325)^{2}\right]^{1 / 2}+1=1.9 \% \mathrm{CS} \\
\mathrm{e}^{-} & =-\left(\left[(\mathrm{RA}+\mathrm{TE})^{2}+\mathrm{SP}^{2}+\mathrm{HE}^{2}+\mathrm{RE}^{2}+\mathrm{PSE}^{2}+\mathrm{SE}^{2}+\mathrm{SMTE}^{2}\right]^{1 / 2}+\mathrm{DR}\right) \\
& =-\left(\left[(0.15+0.666)^{2}+0+0+0+0+0+(0.325)^{2}\right]^{1 / 2}+1\right)=-1.9 \% \mathrm{CS}
\end{aligned}
$$

The error with drift associated with the sensor $\left(\mathrm{e}_{\mathrm{sens}}\right)$ is as follows:

$$
\mathrm{e}_{\text {sens }}= \pm 1.9 \% \mathrm{CS}
$$


Framatome STAR System - Baratron channels

\subsection{Framatome STAR System - Analog Voltage Isolation Module (AVIM)}

\subsubsection{Instrument Data}

Name: AVIM

Manufacturer: Famatome Technologies

Model Number:

Type: Analog Voltage Isolation Module

\subsubsection{Reference Specifications}

Range: 0 to 10 VDC

Resolution:

Accuracy: input to output: $\pm 0.1 \%$ of Full Range (for temperature range 40 to $140^{\circ} \mathrm{F}$ )

Drift: $\pm 0.05 \%$ Full Range

Analog Input/Output: Range: 0.0 VDC to 10.0 VDC

Calibration Interval: 12 months (letter J. Scecina FTI-99-1537)

\subsubsection{Calibration}

A. Assumptions

i.) Turn Down Ratio $=1$

ii.) Calibrations performed at ambient $72 \pm 8^{\circ} \mathrm{F}$ or 64 to $80^{\circ} \mathrm{F}$

iii.) $\mathrm{Min} / \mathrm{max}$ expected temperatures is 40 to $105^{\circ} \mathrm{F}$

iv.) Calibrated Span (CS) $=$ Full Scale $=10$ VDC

v.) Processor Module input error calculation multiplier factor $=1$

B. Channel Uncertainty to MCS - General Service

i.) $\quad \operatorname{Drift}(\mathrm{DR})$

$\mathrm{DR}= \pm 0.05 \% \mathrm{CS}$

ii.) Relative Accuracy (RA)

Given: $\mathrm{RA}=\mathrm{RA}_{\mathrm{avim}}$

$$
= \pm 0.1 \% \text { Full Range }
$$

$\mathrm{RA}= \pm 0.1 \% \mathrm{CS}$

iii.) Static Pressure (SP)

$\mathrm{SP}=0 \% \mathrm{CS}$

Basis: Not a pressure sensor, not subject to the pressure effects of the process

iv.) Humidity Effect (HE)

$\mathrm{HE}=0 \% \mathrm{CS}$

Basis: Housed in NEMA 4 enclosure 
Framatome STAR System - Baratron channels

v.) Temperature Effect (TE)

Given: Reference accuracy is certified for range 40 to $140^{\circ} \mathrm{F}$

And expected range 40 to $105^{\circ} \mathrm{F}$ )

$\mathrm{TE}=0 \% \mathrm{CS}$

vi.) Radiation Effect (RE)

Given: Dose Rate $=8.5 \mathrm{mR} / \mathrm{hr}$

Total Integrated Dose $=8.5 \mathrm{mR} / \mathrm{hr} * 43800 \mathrm{hr}=372,300 \mathrm{mR}$

Radiation Effect Data says there is no effect to $10,000,000 \mathrm{mR}$ TID

Because relatively small dose (based on HNF-SD-SNF-DRD-002, Rev.1)

Assume: $\mathrm{RE}= \pm 0 \% \mathrm{CS}$

vii.) Power Supply Effect (PSE)

Given: No PSE while supply voltage maintained at 15 VDC $\pm 10 \%$

$\mathrm{PSE}=0 \%$

viii.) Seismic Effect (SE)

Device is qualified per IEEE $344-1987$ to withstand $17 \mathrm{~g}$ seismic event

Design Basis event is postulated as $0.26 \mathrm{~g}$

$\mathrm{SE}=0 \%$

ix.) Sensor Measurement and Test Equipment Effect (SMTE)

Test Equipment1: Fluke 743B, DC Volts, Damping is OFF

Calibration Temperature Range with no TempEffect: $>18$ and $<28^{\circ} \mathrm{C}$

Full Scale (FS) $=10 \mathrm{~V}$

$\left(\sim 64\right.$ to $\left.80^{\circ} \mathrm{F}\right)$

Calibrated Span $=0$ to $10 \mathrm{~V}$, Max Reading $(\mathrm{Rdg})=100$ Torr $=10 \mathrm{~V}$

$\mathrm{RA}_{\mathrm{MTE} 1}=0.025 \% * \mathrm{Rdg}+3 *(0.005 \% \mathrm{FS})$ (Given: times 3 with damping OFF)

$=0.025 \% * \mathrm{CS}+0.015 \% \mathrm{FS} *(\mathrm{CS} / \mathrm{FS})$

$= \pm 0.04 \% \mathrm{CS}$

$\mathrm{RA}_{\mathrm{STD} 1}=0.01 \% * \mathrm{Rdg}+3 * 0.005 \% \mathrm{FS}$ (Use given Source Accuracy,

$=0.01 \% * \mathrm{CS}+0.015 \mathrm{FS} *(\mathrm{CS} / \mathrm{FS})$

Assume also *3 with Damping OFF)

$= \pm 0.025 \% \mathrm{CS}$

$\mathrm{RD} 1=0 \% \mathrm{FS}$ (digital readout)

SAl $=0.2 \%$ FS (Assume twice Relative accuracy of AVIM - in/out))

$\mathrm{MTE} 1=\left(\mathrm{RA}_{\mathrm{MTE} 1}{ }^{2}+\mathrm{RA}_{\mathrm{STD1}}{ }^{2}+\mathrm{RD}^{2}+\mathrm{SA}^{2}\right)^{1 / 2}$

MTE1 $=\left(0.04^{2}+0.025^{2}+0^{2}+0.2^{2}\right)^{1 / 2}=0.206 \% \mathrm{CS}$

MTE1 $=0.206 \%$ FS (used for both the input and output of the AVIM.)

$\mathrm{SMTE}=\left(0.206^{2}+0.206^{2}\right)^{1 / 2}=0.291$

x.) STAR AVIM Uncertainties - MCS monitoring

$$
\begin{aligned}
& \mathrm{e}^{+}= {\left[\mathrm{DR}^{2}+\mathrm{RA}^{2}+\mathrm{SP}^{2}+\mathrm{HE}^{2}+\mathrm{TE}^{2}+\mathrm{RE}^{2}+\mathrm{PSE}^{2}+\mathrm{SE}^{2}+\mathrm{SMTE}_{\mathrm{AVIM} \text {-in/out }}\right]^{1 / 2} } \\
& \quad= {\left[0.05^{2}+0.1^{2}+0+0+0+0+0+0+0.291^{2}\right]^{1 / 2}=0.312 \% \mathrm{CS} } \\
& \mathrm{e}_{\mathrm{proc}}= \pm 0.312 \% \mathrm{CS}
\end{aligned}
$$


MCS Modicon- Baratron channels

SNF - 5900 Rev. 0 Page 10 of 31

\subsection{MCS Analog to Digital Module (A/D)}

\subsubsection{Instrument Data}

Name: Analog In Module

Manufacturer: Modicon

Model Number: AVI 03000

Type: 16 bit

2.4.2 Reference Specifications

Range: 0 to $10 \mathrm{VDC}$

Resolution: 16 bit

Accuracy: input to output: $\pm 0.05 \%$ of Full Range

Drift: $\pm 0.0025 \% \mathrm{FS} /{ }^{\circ} \mathrm{C}$

Calibration Interval: 12 months between calibrations (assume)

\subsubsection{Calibration}

A. Assumptions

i.) Calibrations performed at ambient $72 \pm 8^{\circ} \mathrm{F}$ or 64 to $80^{\circ} \mathrm{F}$

ii.) $\mathrm{Min} / \mathrm{max}$ expected temperatures is 40 to $105^{\circ} \mathrm{F}$

iii.) Calibrated Span (CS) $=$ Full Scale $=10 \mathrm{VDC}$

B. Channel Uncertainty
i.) Assume Drift (DR1)
$\mathrm{DR} 1= \pm 0.05 \% \mathrm{CS}$
ii.) Relative Accuracy (RA)
Given: $\mathrm{RA}= \pm 0.05 \% \mathrm{FS}$
$\mathrm{RA}= \pm 0.05 \% \mathrm{CS}$

iii.) Static Pressure (SP)

$\mathrm{SP}=0 \% \mathrm{CS}$

Basis: Not a pressure sensor, not subject to the pressure effects of the process

iv.) Humidity Effect (HE)

$\mathrm{HE}=0 \% \mathrm{CS} \quad$ Basis: Housed in NEMA 4 enclosure

v.) Temperature Effect (TE)

Given: $0.005 \% \mathrm{FS} /{ }^{\circ} \mathrm{C}$

Assume Tmax $=105^{\circ} \mathrm{F}=40.6^{\circ} \mathrm{C}$

Tnorm(lower) $=72-8=64^{\circ} \mathrm{F}=17.8^{\circ} \mathrm{C}$ Lower calibration temperature

Therefore Tdelta $=41 \Delta^{\circ} \mathrm{F}=22.8 \Delta^{\circ} \mathrm{C}$

$\mathrm{TE}=0.005 \% \mathrm{CS} /{ }^{\circ} \mathrm{C} * 22.8 \Delta^{\circ} \mathrm{C}=0.114 \% \mathrm{CS}$ 
MCS Modicon-Baratron channels

vi.) Radiation Effect (RE)

Given: Dose Rate $=8.5 \mathrm{mR} / \mathrm{hr}$

Total Integrated Dose $=8.5 \mathrm{mR} / \mathrm{hr} * 43800 \mathrm{hr}=372,300 \mathrm{mR}$

Radiation Effect Data says there is no effect to $10,000,000 \mathrm{mR}$ TID

Because relatively small dose (based on HNF-SD-SNF-DRD-002, Rev.1)

Assume: $\mathrm{RE}= \pm 0 \% \mathrm{CS}$

vii.) Power Supply Effect (PSE)

Given: External power not required

PSE $=0 \%$

viii.) Seismic Effect (SE)

No credit for this device is taken during or after a seismic event.

$\mathrm{SE}=0 \%$

ix.) Sensor Measurement and Test Equipment Effect (SMTE)

Test Equipment1: Fluke 743B, DC Volts, 5 digit display

See Section 2.3.3 B.ix.)

SMTE $=$ MTE $1=0.206 \% \mathrm{CS}$

x.) Total MCS A/D Uncertainties

$$
\begin{aligned}
\mathrm{e}^{+} & =\left[\mathrm{DR}^{2}+\mathrm{RA}^{2}+\mathrm{SP}^{2}+\mathrm{HE}^{2}+\mathrm{TE}^{2}+\mathrm{RE}^{2}+\mathrm{PSE}^{2}+\mathrm{SE}^{2}+\mathrm{SMTE}^{2}\right]^{1 / 2} \\
& =\left[0.05^{2}+0.05^{2}+0+0+0.114^{2}+0+0+0+0.206^{2}\right]^{1 / 2}=0.246 \% \mathrm{CS} \\
\mathrm{e}_{\mathrm{a} / \mathrm{d}} & = \pm 0.246 \% \mathrm{CS}
\end{aligned}
$$


OCRWM Loop Uncertainty- Baratron channels

\subsection{Total Loop Uncertainty - General Service MCS Loop}

$$
\begin{aligned}
& \mathrm{CU}^{+}=\left[\mathrm{e}_{\text {sens-dr }}^{2}+\mathrm{e}_{\text {proc }}^{2}+\mathrm{e}_{\mathrm{a} / \mathrm{d}}^{2}\right]^{1 / 2}+\mathrm{DR}_{\text {sens }}+\mathrm{B}_{\text {proc }} \\
& \mathrm{CU}^{+}{ }_{\mathrm{mcs}}= \pm\left\{\left[0.9^{2}+0.312^{2}+0.25^{2}\right]^{1 / 2}+1+0\right\}=1.985 \% \mathrm{CS} \\
& \mathrm{CU}_{\mathrm{mcs}}= \pm 1.985 \% \mathrm{CS}
\end{aligned}
$$

\subsection{MCS Vacuum Loop Error Determination (OCRWM)}

$\mathrm{E}_{\mathrm{ocrwm}}= \pm 1.985 \% \mathrm{CS} * 100$ torr $/ \mathrm{CS}+2$ torr (system drop) $= \pm 3.99$ torr 
Delta Pressure - Baratron channels

\subsection{Delta Pressure Accuracy}

2.7.1 Uncertainty Terms that Affect Accuracy

Note: Since this is a Delta Pressure over a relatively short time, several terms are no longer necessary. However, since the accuracy test could be run on a different day than when the result may be required, temperature does affect the results.

A. Sensor Uncertainty

i.) Relative Accuracy (RA)

Given: $\mathrm{RA}=0.15 \%$ Reading (Rdg)

ii.) $\quad$ Drift (DR)

Assume DR $=0$ for time interval of 1 hour

iii.) Temperature Effect (TE)

Given: $\mathrm{TE}_{\mathrm{s}}= \pm 0.020 \% \mathrm{Rdg} /{ }^{\circ} \mathrm{C}$ for $\operatorname{Span}(100 \mathrm{Torr})$

$\mathrm{TE}_{\mathrm{z}}= \pm 0.007 \% \mathrm{FS} /{ }^{\circ} \mathrm{C}$ for Zero (0 Torr)

Uncertainty below temp range of $15^{\circ} \mathrm{C}\left(40\right.$ to $\left.60^{\circ} \mathrm{F}\right)= \pm 0.05$ torr

With $\mathrm{TDR}=1, \mathrm{CS}=\mathrm{FS}=\mathrm{Span}=100$ Torr

Temp Effect $=0.02 \% * \mathrm{Rdg} /{ }^{\circ} \mathrm{C}+\left(0.007 \% * \mathrm{FS} /{ }^{\circ} \mathrm{C}\right)$

Temp Uncertainty $=0.0002 * \mathrm{Rdg} /{ }^{\circ} \mathrm{C}+\left(0.007\right.$ torr $/{ }^{\circ} \mathrm{C}+0.05$ torr $)$

Assume Tmax $=105^{\circ} \mathrm{F}$, Tnorm (lower) $=72-8=64^{\circ} \mathrm{F}$

Therefore Tdelta $=105-64=41 \Delta^{\circ} \mathrm{F}=22.8 \Delta^{\circ} \mathrm{C}$

Temp Uncertainty $=0.0002 * 22.8 * \mathrm{Rdg}+(0.007 * 22.8$ torr +0.05 torr $)$

$$
=0.00456 * \mathrm{Rdg}+(0.21 \text { torr })
$$

NOTE: Assume that the 0.21 torr (the Zero Drift and the uncertainty below temperature range) is a bias and can be ignored over the time interval

Temp Uncertainty $=0.00456 *$ Rdg torr

iv.) Framatome Analog Voltage Isolation Module (AVIM) Input and Output Error

$\mathrm{RA}_{\mathrm{F}}= \pm 0.1 \%$ of Full Range

Full Range $=10$ VDC $\Leftrightarrow=>100$ torr

$\mathrm{RA}_{\mathrm{F}}=\left(0.1^{2}\right)^{1 / 2}=0.1 \% \mathrm{CS}=0.1 \% 10 \mathrm{~V} / 100 \% * 100$ torr $/ 10 \mathrm{~V}=0.1$ torr

v.) MCS Input and Output Error

Processor Module input error calculation multiplier factor $\beta=1.0$

MCS 16 bit Accuracy: $\pm 0.05 \%$ of Full Range (Analog Input)

Full Range $=10 \mathrm{VDC} \Leftrightarrow=100$ torr

$\mathrm{RA}_{\mathrm{mcs}}=\left(\beta * \mathrm{RA}_{\mathrm{I}}^{2}\right)^{1 / 2}$

$\mathrm{RA}_{\text {mcs }}=\left(0.05^{2}\right)^{1 / 2}=0.05 \% \mathrm{CS}=0.05 \% 10 \mathrm{~V} / 100 \% * 100$ torr $/ 10 \mathrm{~V}=0.05$ torr 
Delta Pressure - Baratron channels

vi.) Total Sensor Delta Pressure Uncertainties

Sensor and Readout Error on Span Measurements

Pressure rise from 0.5 to 2.9 torr

$$
\begin{aligned}
\mathrm{SE}_{1} & = \pm\left[\mathrm{RA}_{\mathrm{s}}{ }^{2}+\mathrm{TE}_{\mathrm{s}}{ }^{2}+\mathrm{RA}_{\mathrm{F}}{ }^{2}+\mathrm{RA}_{\mathrm{mcs}}{ }^{2}\right]^{1 / 2} \\
& = \pm\left[\left(0.0015^{*} 0.5\right)^{2}+\left(0.00456^{*} 0.5\right)^{2}+0.1^{2}+0.05^{2}\right]^{1 / 2} \\
& = \pm 0.112 \text { torr } \\
\mathrm{SE}_{2} & = \pm\left[\mathrm{RA}_{\mathrm{s}}{ }^{2}+\mathrm{TE}_{\mathrm{s}}{ }^{2}+\mathrm{RA}_{\mathrm{F}}{ }^{2}+\mathrm{RA}_{\mathrm{mcs}}{ }^{2}\right]^{1 / 2} \\
& = \pm\left(\left[\left(0.0015^{*} 2.9\right)^{2}+\left(0.00456^{*} 2.9\right)^{2}+0.1^{2}+0.05^{2}\right]^{1 / 2}\right. \\
& = \pm 0.113 \text { torr }
\end{aligned}
$$

Pressure rise from 5 to 7.4 torr

$$
\begin{aligned}
\mathrm{SE}_{1} & = \pm\left[\mathrm{RA}_{\mathrm{s}}^{2}+\mathrm{TE}_{\mathrm{s}}{ }^{2}+\mathrm{RA}_{\mathrm{F}}^{2}+\mathrm{RA}_{\mathrm{mcs}}^{2}\right]^{1 / 2} \\
& = \pm\left[\left(0.0015^{*} 5.0\right)^{2}+\left(0.00456^{*} 5\right)^{2}+0.1^{2}+0.05^{2}\right]^{1 / 2} \\
& = \pm 0.114 \text { torr } \\
\mathrm{SE}_{2} & = \pm\left[\mathrm{RA}_{\mathrm{s}}{ }^{2}+\mathrm{TE}_{\mathrm{s}}{ }^{2}+\mathrm{RA}_{\mathrm{F}}{ }^{2}+\mathrm{RA}_{\mathrm{mss}}{ }^{2}\right]^{1 / 2} \\
& = \pm\left[\left(0.0015^{*} 7.4\right)^{2}+\left(0.00456^{*} 7.4\right)^{2}+0.1^{2}+0.05^{2}\right]^{1 / 2} \\
& = \pm 0.114 \text { torr }
\end{aligned}
$$

\subsubsection{MCS Delta Vacuum Loop Error Determination (OCRWM)}

Without considering Temperature Effects:

For a pressure rise test from 0.5 torr to 2.9 torr or from 5.0 torr to 7.4 torr, the OCRWM loop error on each measurement is less than or equal to \pm 0.114 torr. Since it is an \pm error, the maximum error would be 0.228 torr.

i.e.) $0.5 \pm 0.114$ to $2.9 \pm 0.114$ becomes 0.386 to 3.014 or 0.614 to 2.786

$$
\mathrm{Span}=2.9( \pm 0.114)-0.5( \pm 0.114)=2.4 \pm 0.228 \text { torr }
$$

Considering Temperature Effects:

When temperature variation (i.e., the potential difference between calibration temperature and test temperature) is included, the loop error on all measurements $\leq 8$ torr ( $12-4$ torr measurement error) is $\leq 0.118$ torr. Since it is an \pm error, the maximum error would be 0.236 torr.

The maximum resolution is 0.01 torr. Therefore, rounding up to two digit accuracy,

$$
\text { DELTA } E_{\text {ocrwm }}= \pm 0.24 \text { torr }
$$


Calibration Requirements - Baratron channels

\subsection{OCRWM Vacuum Pressure Calibration Requirements}

\subsubsection{Vacuum Pressure Sensor/Loop}

\subsubsection{MKS Baratron}

A. Assumptions

i.) Turn Down Ratio $=1$ (transmitter will have a full span of 100 Torr)

ii.) Calibrations performed at ambient $72 \pm 8^{\circ} \mathrm{F}$ or 64 to $80^{\circ} \mathrm{F}$

B. Sensor Uncertainty

i.) Relative Accuracy (RA)

Given: $\mathrm{RA}=0.15 \%$ Reading (Rdg)

C. Tolerance Requirements

i.) Proof Mode and Pressure Test (Range $\leq 10$ Torr)

$\pm 0.15 \%$ Reading $* 10$ Torr $= \pm 0.015$ Torr

Resolution $=0.01$ Torr

Therefore, rounding up

Tolerance $($ Range $\leq 10$ Torr) $= \pm 0.02$

ii.) Pressure Test (Range $>10$ Torr)

$\pm 0.15 \% \mathrm{CS} * 100 \mathrm{Torr} / \mathrm{CS}=0.15$ Torr

Tolerance (Range 10 to 100 Torr $)= \pm 0.15$ Torr

D. OCRWM Sensor Calibration Tolerance

RANGE

0 to 10 Torr

10 to 100 Torr
Sensor Tolerance

\pm 0.02 torr

\pm 0.15 torr 
Calibration Requirements - Baratron channels

SNF - 5900 Rev. 0 Page 16 of 31

\subsubsection{Loop Components}

2.8.1.2.1 Framatome STAR System - Analog Voltage Isolation Module (AVIM)

A. Assumptions

i.) Calibrations performed at ambient $72 \pm 8^{\circ} \mathrm{F}$ or 64 to $80^{\circ} \mathrm{F}$

ii.) Calibrated Span (CS) $=$ Full Scale (FS) $=10 \mathrm{VDC}$

B. Channel Uncertainty to MCS - General Service

i.) Relative Accuracy (RA)

Given: $\mathrm{RA}=\mathrm{RA}_{\mathrm{avim}}= \pm 0.1 \%$ Full Range

$\mathrm{RA}= \pm 0.1 \% \mathrm{CS}$

2.8.1.2.2 Modicon Analog to Digital Module (A/D)

A. Assumptions

i.) Calibrations performed at ambient $72 \pm 8^{\circ} \mathrm{F}$ or 64 to $80^{\circ} \mathrm{F}$

ii.) Calibrated Span (CS) $=$ Full Scale (FS) $=10 \mathrm{VDC}$

B. Channel Uncertainty

i.) Relative Accuracy (RA)

Given: $\mathrm{RA}= \pm 0.05 \%$ FS

\subsection{OCRWM Loop Calibration}

A. Loop Tolerance (LT) Determination

Range $\leq 10$ Torr, Max Reading $=10$ Torr, $\mathrm{FS}=\mathrm{CS}=100$ Torr

$$
\begin{aligned}
\mathrm{LT} & = \pm\left[\mathrm{RA}_{\mathrm{s}}{ }^{2}+\mathrm{RA}_{\mathrm{F}}{ }^{2}+\mathrm{RA}_{\mathrm{mcs}}{ }^{2}\right]^{1 / 2}= \pm\left[(0.15 \% \mathrm{Rdg})^{2}+0.1 \% \mathrm{FS}^{2}+0.05 \% \mathrm{FS}^{2}\right]^{1 / 2} \\
& = \pm\left[\left(0.0015^{*} 10\right)^{2}+(0.1 \% * 100 \mathrm{Torr} / 100 \%)^{2}+(0.05 \% * 100 \mathrm{Torr} / 100 \%)^{2}\right]^{1 / 2} \\
\mathrm{LT} & = \pm\left[\left(0.0015^{*} 10\right)^{2}+0.1^{2}+0.05^{2}\right]^{1 / 2} \text { Torr } \\
\mathrm{LT} & = \pm 0.11 \text { Torr (Note Resolution is } 0.01, \text { Truncate to } 0.1 \text { Torr) } \\
\mathrm{LT} & = \pm 0.1 \text { Torr }
\end{aligned}
$$

Range 10 to 100 Torr, Max Reading $=\mathrm{FS}=\mathrm{CS}=100$ Torr

$$
\begin{aligned}
\mathrm{LT} & = \pm\left[\mathrm{RA}_{\mathrm{s}}{ }^{2}+\mathrm{RA}_{\mathrm{F}}{ }^{2}+\mathrm{RA}_{\mathrm{mcs}}{ }^{2}\right]^{1 / 2} \\
& = \pm\left[0.15^{2}+0.1^{2}+0.05^{2}\right]^{1 / 2} \% \mathrm{CS}= \pm 0.19 \% \mathrm{CS} \\
& = \pm 0.19 \% \mathrm{CS} * 100 \mathrm{Torr} / 100 \% \mathrm{CS}=0.19 \mathrm{Torr}
\end{aligned}
$$

Rounding $0.19 \approx 0.2$

B. OCRWM Loop Calibration Tolerance

\begin{tabular}{ll} 
RANGE & Loop Tolerance \\
\hline$\leq 10$ Torr & \pm 0.1 torr \\
10 to 100 Torr & \pm 0.2 torr
\end{tabular}


Vacuum Pressure M\&TE Requirements

\subsubsection{Measurement and Test Equipment}

i.) Test Equipment Error Maximum (TEM)

1. Test Equipment1: Volt Meter

Assume Fluke 743B, DC Volts with Damping OFF or equivalent

Calibration Temperature Range with no TempEffect: $>18$ and $<28^{\circ} \mathrm{C}$

$\left(\sim 64\right.$ to $\left.80^{\circ} \mathrm{F}\right)$

$$
\begin{aligned}
\mathrm{RA}_{\mathrm{MTE} 1} & =0.025 \% * \mathrm{Rdg}+3 *(0.005 \% \mathrm{FS})(\text { Given: times } 3 \text { with damping OFF) } \\
& =0.025 \% * \mathrm{CS}+0.015 \% \mathrm{FS}(\mathrm{CS} / \mathrm{FS}) \\
& = \pm 0.04 \% \mathrm{CS} \\
\mathrm{RA}_{\mathrm{STD} 1} & =0.01 \% * \mathrm{Rdg}+3 * 0.005 \% \mathrm{FS} \text { (Use given Source Accuracy, } \\
& =0.01 \% * \mathrm{CS}+0.015 \mathrm{FS} *(\mathrm{CS} / \mathrm{FS}) \\
& = \pm 0.025 \% \mathrm{CS} \\
\mathrm{RD} 1= & 0 \% \mathrm{FS} \text { (digital readout) }
\end{aligned}
$$

TEM Contribution from Volt Meter

Note: Setting Accuracy (SA) is not considered.

$\mathrm{TEM}= \pm\left(0.04^{2}+0.025^{2}+0\right)^{1 / 2}= \pm 0.05 \% \mathrm{CS}$

\section{Test Equipment2, Vacuum Standard}

Assume MKS Portable Pressure Transfer Standard Type PBTS1 or equivalent Operating Temperature Range: 15 to $40^{\circ} \mathrm{C}$

Full Scale (FS) $=100$ Torr

Resolution $=0.0001$ Torr

$\mathrm{RA}_{\mathrm{MTE} 2}=0.05 \% * \mathrm{Rdg}$

$\mathrm{RA}_{\mathrm{STD} 2}<=0.01 \% \mathrm{CS}$ (Assume Calibration Standard used was at least $4: 1$ )

$\mathrm{RD} 2=0 \% \mathrm{FS}$ (digital readout)

TEM Contribution from Pressure Transfer Standard

$\mathrm{TEM}= \pm\left(0.05^{2}+0.01^{2}+0\right)^{1 / 2}= \pm 0.05 \% \mathrm{CS}$ 
Rosemount Temperature Transmitter

\section{MCO temperature transmitter to MCS}

Figure 3.1 TW Temperature Simplified Block Diagram

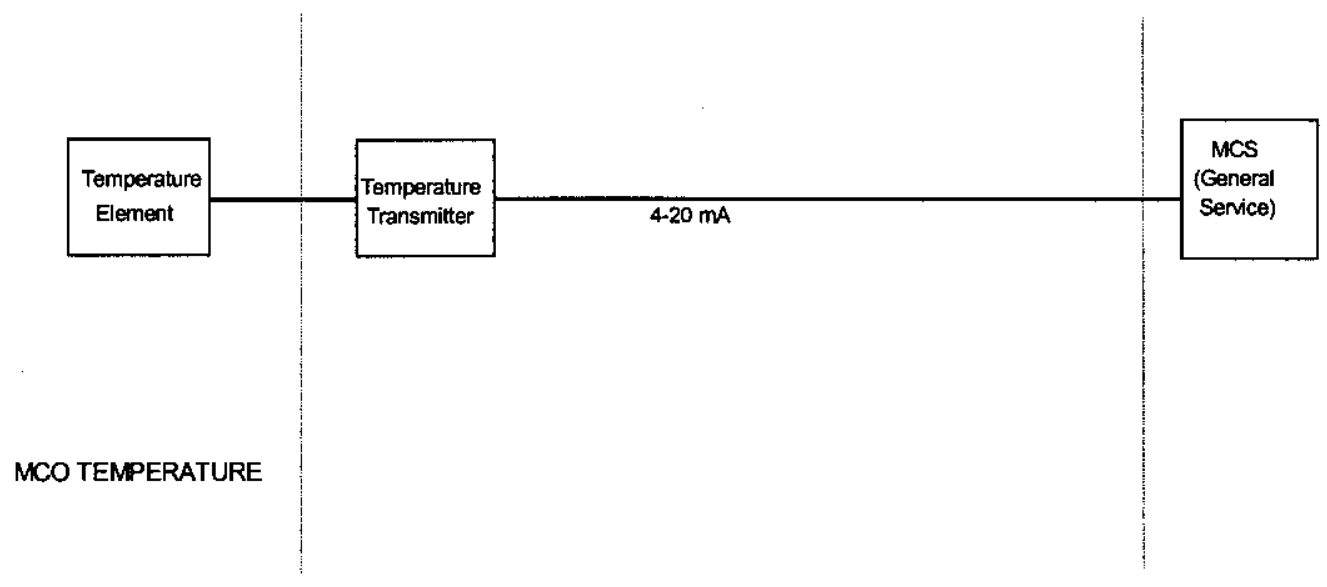

\subsection{Plant Operating Conditions}

The tempered water (TW) water temperature, in combination with the MCO absolute pressure, provides an indication of water content in the MCO. This information on Proof of Drying is required for the Office of Civilian Radioactive Waste Management (OCRWM).

\subsubsection{Drying Mode Operations}

A period of time under vacuum (Proof Mode) is completed at the CVD Facility to assure free water has been sufficiently removed from the MCO. This operation will measure the MCO internal pressure above a specific temperature for a specified time.

- Proof Mode: the $\mathrm{MCO}$ must be $\geq 40^{\circ} \mathrm{C}$, and the $\mathrm{MCO}$ pressure must be $\leq 12$ torr for a total time of greater or equal to

(1) 8 hours for an MCO with no scrap baskets,

(2) 20 hours for an MCO with one scrap basket,

(3) 28 hours for an MCO with two scrap baskets.

- The final pressure rebound test: the MCO must be $\geq 40{ }^{\circ} \mathrm{C}$, and the $\mathrm{MCO}$ evacuated to $\leq 10$ torr. $\mathrm{MCO}$ pressure rise shall be $\leq 2.4$ torr per hour for a duration of $\geq 1$ hour.

This information, and the analysis that documents the correlation of temperature, pressure, and time to water content in the MCO, will be used to document the fuel in the MCO has met the "no free liquid" criteria. 
Rosemount Temperature Transmitter

\subsection{Temperature Transmitter $($ TIT $-3 * 05$, TIT $-3 * 12)$}

\subsubsection{Instrument Data}

Name: Smart Temperature Transmitters

Manufacturer: Rosemount Instruments

Model Number: 3244MV 1 NA M5 C2 Q4

Type: Model 3244 MV Smart Temperature Transmitter

\subsubsection{Reference Specifications}

\subsubsection{Transmitter Reference Specifications}

$\begin{array}{ll}\text { Temperature Range: } & 0 \text { to } 100^{\circ} \mathrm{C} \text { span } \\ \text { Ambient Temp Effect: } & \text { Digital:0.0015 } \\ & \text { D/A: } 0.001 \% \text { span per } 1{ }^{\circ} \mathrm{C} \text { change in ambient } \\ & \text { When the location has range between }-40 \text { to } 85^{\circ} \mathrm{C} \\ & \pm 0.1 \% \mathrm{Rdg} \text { or } 0.1^{\circ} \mathrm{C} \text { whichever is greater } \\ \text { Stability: } & \text { digital: } \pm 0.14^{\circ} \mathrm{C}, \mathrm{D} / \mathrm{A}: \pm 0.02 \% \text { of span } \\ \text { Accuracy: } & \pm 0.05^{\circ} \mathrm{C} \\ \text { Repeatability: } & 2 \text { years between calibrations }\end{array}$

\subsubsection{RTD Reference Specifications}

$\begin{array}{ll}\text { Input Temp Range: } & 0 \text { to } 100{ }^{\circ} \mathrm{C} \\ \text { Stability: } & \pm 0.08 \% \text { ice point resistance shift } \\ \text { Repeatability: } & \pm 0.05 \% \\ \text { Calibration Interval: } & 2 \text { years between calibrations }\end{array}$

\subsubsection{Calibration}

A. Assumptions
i.) Transmitter calibrations performed at ambient $72 \pm 8^{\circ} \mathrm{F}$ or 64 to $80^{\circ} \mathrm{F}$
ii.) $\mathrm{Min} / \mathrm{max}$ expected normal ambient temperature range is 40 to $105^{\circ} \mathrm{F}$
iii.) Calibration Interval $=1$ year

B. Sensor Uncertainty

i.) Relative Accuracy (RA)

Given: transmitter $\mathrm{RA}=0.14{ }^{\circ} \mathrm{C} *\left(100 \% \mathrm{CS} / 100^{\circ} \mathrm{C}\right)+0.02 \% \mathrm{CS}=0.16 \% \mathrm{CS}$

Given: sensor Repeatability $=0.05 \%$ Span

$$
\mathrm{RA}=\left(0.16^{2}+0.05^{2}\right)^{1 / 2}=0.17 \% \mathrm{CS}
$$


Rosemount Temperature Transmitter

ii.) Drift

Assume RTD Drift is the ice point resistance shift (zero) $0.08 \% \mathrm{CS}$

Treat as bias. i.e., additive

$\mathrm{DR}= \pm(0.1 \% \mathrm{CS}+0.08 \% \mathrm{CS})= \pm 0.18 \% \mathrm{CS}$

iii.) Temperature Effect (TE)

Given: Transmitter TE $=0.0015^{\circ} \mathrm{C} /\left(1^{\circ} \mathrm{C}\right.$ change in ambient $) *$ Tdelta

$+0.001 \% \mathrm{CS} /\left(1^{\circ} \mathrm{C}\right.$ change in ambient $) *$ Tdelta

Assume $\operatorname{Tmax}=105^{\circ} \mathrm{F}=40.6^{\circ} \mathrm{C}$

Tnorm(lower) $=72-8=64^{\circ} \mathrm{F}=17.8^{\circ} \mathrm{C}$ Lower calibration temperature

Therefore Tdelta $=105-64=41 \Delta^{\circ} \mathrm{F}=22.8 \Delta^{\circ} \mathrm{C}$

Transmitter Temperature Effect

$\mathrm{TE}=0.0015^{\circ} \mathrm{C} / 1^{\circ} \mathrm{C} * 22.8^{\circ} \mathrm{C} *\left(100 \% \mathrm{CS} / 100^{\circ} \mathrm{C}\right)+0.001 \% \mathrm{CS} / 1^{\circ} \mathrm{C} * 22.8^{\circ} \mathrm{C}$

$\mathrm{TE}=0.057 \% \mathrm{CS}$

iv.) Static Pressure (SP)

$\mathrm{SP}=0 \%$

Basis: Assumed zero for low pressures.

v.) Humidity Effect (HE)

$\mathrm{HE}=0 \%$

Basis: Active portions are sealed i.e. not exposed to outside environment.

NEMA-4X Enclosure. Listed range 0 to $100 \%$ relative humidity

vi.) Radiation Effect (RE)

Given: Dose Rate $=8.5 \mathrm{mR} / \mathrm{hr}$

Total Integrated Dose $=8.5 \mathrm{mR} / \mathrm{hr} * 43800 \mathrm{hr}=372,300 \mathrm{mR}$ TID

No Radiation Effect Data, RE is assumed zero because of the very low dose

Assume: $\mathrm{RE}= \pm 0 \%$

vii.) Power Supply Effect (PSE)

Given: PSE $= \pm 0.005 \%$ of span $/$ volt

Assume power supply variance $15 \mathrm{VDC} \pm 10 \%=1.5 \mathrm{~V}$

$\mathrm{PSE}= \pm 0.005 \%$ of $\mathrm{span} / \mathrm{V} * 1.5 \mathrm{~V}=0.0075 \% \mathrm{CS}$

viii.) Seismic Effect (SE)

No credit for this device is taken during or after a seismic event, unit will have to be re-qualified and recalibrated if seismic level exceeds TBD $\mathrm{g}$.

$\mathrm{SE}=0 \%$ 
Rosemount Temperature Transmitter

ix.) Sensor Measurement and Test Equipment Effect (SMTE)

Test Equipment 1: Fluke 87, DC voltage mode with a 4 digit display

$\mathrm{RA}_{\mathrm{MTE} 1}=0.05 \% \mathrm{Rdg}+1 \mathrm{digit}$

Max Reading $=15 \mathrm{~V}=\mathrm{CS}$

$\mathrm{RA}_{\text {MTE } 1}= \pm 0.05 \% * 15 \mathrm{~V} /(15 \mathrm{~V} / \mathrm{CS})+0.01 \mathrm{~V} /(15 \mathrm{~V} / \mathrm{CS}) * 100 \%$ $= \pm 0.12 \% \mathrm{CS}$

$\mathrm{RA}_{\mathrm{STD} 1}=0.04 \%$ (assume 4 times more accurate)

$\mathrm{RD} 1=0 \%$ (digital read out)

$\mathrm{SA} 1=0.25 \%$ (assume setting accuracy equal to twice the relative accuracy)

$\mathrm{MTE} 1=\left(\mathrm{RA}_{\mathrm{MTE1}}{ }^{2}+\mathrm{RA}_{\mathrm{STDI}}{ }^{2}+\mathrm{RD} 1^{2}+\mathrm{SA} 1^{2}\right)^{1 / 2}$ $=\left(0.12^{2}+0.04^{2}+0^{2}+0.25^{2}\right)^{1 / 2}$

MTE1 $=0.28 \% \mathrm{CS}$

Test Equipment2: NIST traceable Temperature Standard AMETEK 140SE JOFRA dry block

Accuracy $= \pm 0.3^{\circ} \mathrm{C}$

Stability $= \pm 0.05^{\circ} \mathrm{C}$

$\mathrm{RA}_{\mathrm{MTE1}}=$ Accuracy + Stability

$\mathrm{RA}_{\mathrm{MTEl}}=0.3+0.05=0.35^{\circ} \mathrm{C}=0.35 / 1.0 \mathrm{CS}=0.35 \% \mathrm{CS}$

$\mathrm{RA}_{\mathrm{MTE} 1}= \pm 0.35 \% \mathrm{CS}$

$\mathrm{RA}_{\mathrm{STD} 1}= \pm 0.1^{\circ} \mathrm{C}$ (NIST standard (RTD used for calibration)

$\mathrm{RA}_{\mathrm{STD} 1}=0.1^{\circ} \mathrm{C}(100 / 100)=0.1 \% \mathrm{CS}$

$\mathrm{RD} 1=0 \% \mathrm{CS}$ (digital readout)

$\mathrm{SA} 1=0.1 \% \mathrm{CS}$

(Assume standard temperature setpoint accuracy equal to relative accuracy)

$$
\begin{aligned}
& \text { MTE1 }=\left(\mathrm{RA}_{\mathrm{MTE}}{ }^{2}+\mathrm{RA}_{\mathrm{STD1}}{ }^{2}+\mathrm{RD}^{2}+\mathrm{SA}^{2}\right)^{1 / 2} \\
& \text { MTE1 }=\left(0.35^{2}+0.1^{2}+0+0.1^{2}\right)^{1 / 2}=0.4 \% \mathrm{CS} \\
& \mathrm{SMTE}=\left(\mathrm{MTE}^{2}+\mathrm{MTE}^{2}\right)^{1 / 2}=\left(0.28^{2}+0.4^{2}\right)^{1 / 2} \% \mathrm{CS}=0.49 \% \mathrm{CS}
\end{aligned}
$$

x.) Total Uncertainties

$$
\begin{aligned}
& \mathrm{e}^{+}=\left[\mathrm{RA}^{2}+\mathrm{DR}^{2}+\mathrm{TE}^{2}+\mathrm{SP}^{2}+\mathrm{HE}^{2}+\mathrm{RE}^{2}+\mathrm{PSE}^{2}+\mathrm{SE}^{2}+\mathrm{SMTE}^{2}\right]^{1 / 2}+\text { bias } \\
& =\left[0.17^{2}+0.1^{2}+0.057^{2}+0+0+0+0.0075^{2}+0+0.49^{2}\right]^{1 / 2}+0.08 \\
& =(0.53+0.08)=0.61 \% \mathrm{CS} \\
& \mathrm{e}^{-}=-\left\{\left[\mathrm{RA}^{2}+\mathrm{DR}^{2}+\mathrm{TE}^{2}+\mathrm{SP}^{2}+\mathrm{HE}^{2}+\mathrm{RE}^{2}+\mathrm{PSE}^{2}+\mathrm{SE}^{2}+\mathrm{SMTE}^{2}\right]^{1 / 2}+\mathrm{bias}\right\} \\
& =-\left\{\left[0.17^{2}+0.1^{2}+0.057^{2}+0+0+0+0.0075^{2}+0+0.49^{2}\right]^{1 / 2}+0.08\right\} \\
& =-0.61 \% \mathrm{CS} \\
& \mathrm{e}_{\mathrm{sens}}= \pm 0.61 \% \mathrm{CS}
\end{aligned}
$$


MCS Modicon - Rosemount Temperature Channels

\subsection{MCS Analog to Digital Module (A/D)}

\subsubsection{Instrument Data}

Name: Analog In Module

Manufacturer: MODICON

Model Number: ACI 03000

Type: 12 bit

\subsubsection{Reference Specifications}

Range: 4 to $20 \mathrm{~mA}$

Resolution: 12 bit

Accuracy: input to output: $\pm 0.1 \%$ of Full Range

Drift: $\pm 0.0025 \% \mathrm{FS} /{ }^{\circ} \mathrm{C}$

Calibration Interval: 12 months between calibrations (assume)

\subsubsection{Calibration}

A. Assumptions

i.) Calibrations performed at ambient $72 \pm 8^{\circ} \mathrm{F}$ or 64 to $80^{\circ} \mathrm{F}$

ii.) $\mathrm{Min} / \mathrm{max}$ expected temperatures is 40 to $95^{\circ} \mathrm{F}$

iii.) Calibrated Span (CS) $=$ Full Scale $=10 \mathrm{VDC}$

B. Channel Uncertainty
i.) Assume Drift (DR1)
$\mathrm{DR} 1= \pm 0.05 \% \mathrm{CS}$
ii.) Relative Accuracy (RA)
Given: $\mathrm{RA}= \pm 0.1 \% \mathrm{FS}$ (worst case)
$\mathrm{RA}= \pm 0.1 \% \mathrm{CS}$

iii.) Static Pressure (SP)

$$
\mathrm{SP}=0 \% \mathrm{CS}
$$

Basis: Not a pressure sensor, not subject to the pressure effects of the process

iv.) Humidity Effect (HE)

$$
\mathrm{HE}=0 \% \mathrm{CS}
$$

Basis: Housed in NEMA 4 enclosure

v.) Temperature Effect (TE)

Given: $0.005 \% \mathrm{FS} /{ }^{\circ} \mathrm{C}$

Assume Tmax $=105^{\circ} \mathrm{F}=40.6^{\circ} \mathrm{C}$

Tnorm (lower) $=72-8=64^{\circ} \mathrm{F}=17.8^{\circ} \mathrm{C}$ Lower calibration temperature

Therefore Tdelta $=105-64=41 \Delta^{\circ} \mathrm{F}=22.8 \Delta^{\circ} \mathrm{C}$

$\mathrm{TE}=0.005 \% \mathrm{CS} /{ }^{\circ} \mathrm{C} * 22.8 \Delta^{\circ} \mathrm{C}=0.114 \% \mathrm{CS}$ 
Calibration Requirements- Rosemount Temperature Channels

vi.) Radiation Effect (RE)

Given: Dose Rate $=8.5 \mathrm{mR} / \mathrm{hr}$

Total Integrated Dose $=8.5 \mathrm{mR} / \mathrm{hr} * 43800 \mathrm{hr}=372,300 \mathrm{mR}$

Radiation Effect Data says there is no effect to $10,000,000 \mathrm{mR}$ TID

Because relatively small dose (based on HNF-SD-SNF-DRD-002, Rev.1)

Assume: $\mathrm{RE}= \pm 0 \% \mathrm{CS}$

vii.) Power Supply Effect (PSE)

Given: External power not required

PSE $=0 \%$

viii.) Seismic Effect (SE)

No credit for this device is taken during or after a seismic event.

$\mathrm{SE}=0 \%$

ix.) Sensor Measurement and Test Equipment Effect (SMTE)

Test Equipment 1: Fluke 87, DC voltage mode with a 4 digit display

See Section 3.2.3 B. ix)

$\mathrm{MTE} 1=0.28 \% \mathrm{CS}$

$\mathrm{SMTE}=\mathrm{MTE} 1=0.28 \% \mathrm{CS}$

x.) Total MCS A/D Uncertainties

$$
\begin{aligned}
\mathrm{e}^{+} & =\left[\mathrm{DR}^{2}+\mathrm{RA}^{2}+\mathrm{SP}^{2}+\mathrm{HE}^{2}+\mathrm{TE}^{2}+\mathrm{RE}^{2}+\mathrm{PSE}^{2}+\mathrm{SE}^{2}+\mathrm{SMTE}^{2}\right]^{1 / 2} \\
& =\left[0.05^{2}+0.1^{2}+0+0+0.114^{2}+0+0+0+0.28^{2}\right]^{1 / 2}=0.322 \% \mathrm{CS} \\
\mathrm{e}_{\mathrm{a} / \mathrm{d}} & = \pm 0.322 \% \mathrm{CS}
\end{aligned}
$$

\subsection{Total Loop Uncertainty - General Service MCS Loop}

$\mathrm{CU}^{+}=\left[\mathrm{e}^{2}{ }_{\text {sens-dr }}+\mathrm{e}_{\mathrm{a} / \mathrm{d}}^{2}\right]^{1 / 2}+\mathrm{B}_{\text {sens }}+\mathrm{B}_{\text {proc }}$

$\mathrm{CU}_{\mathrm{mcs}}= \pm\left\{\left[0.53^{2}+0.322^{2}\right]^{1 / 2}+0.08+0\right\}=0.62+0.08=0.70 \% \mathrm{CS}$

$\mathrm{CU}_{\mathrm{mcs}}= \pm 0.70 \% \mathrm{CS}$

\subsection{MCS TW Temperature Loop Error Determination (OCRWM)}

$\mathrm{E}_{\text {ocrwm }}= \pm 0.70 \% \mathrm{CS} * 100^{\circ} \mathrm{C} / \mathrm{CS}= \pm 0.70^{\circ} \mathrm{C}$ 
Calibration Requirements- Rosemount Temperature Channels

\subsection{Calibration Requirements}

3.6.1 Annulus Temperature Sensor/Loop

3.6.1.1 Rosemount Temperature Transmitter

A. Assumptions

i.) Transmitter calibrations performed at ambient $72 \pm 8^{\circ} \mathrm{F}$ or 64 to $80^{\circ} \mathrm{F}$

ii.) Calibration Interval $=1$ year

B. Sensor Uncertainty

i.) Relative Accuracy (RA)

Given: transmitter $\mathrm{RA}=0.14{ }^{\circ} \mathrm{C} *\left(100 \% \mathrm{CS} / 100^{\circ} \mathrm{C}\right)+0.02 \% \mathrm{CS}=0.16 \% \mathrm{CS}$

Given: sensor Repeatability $=0.05 \%$ Span

$\mathrm{RA}=\left(0.16^{2}+0.05^{2}\right)^{1 / 2}=0.17 \% \mathrm{CS}=0.17 \% * 100{ }^{\circ} \mathrm{C} / 100= \pm 0.17^{\circ} \mathrm{C}$

C. OCRWM Sensor Calibration Tolerance

RANGE Sensor Tolerance

0 to $100^{\circ} \mathrm{C} \quad \pm 0.17^{\circ} \mathrm{C}$

\subsubsection{Loop Components}

3.6.1.2.1 Framatome STAR System - Analog Voltage Isolation Module (AVIM)

A. Assumptions

i.) Calibrations performed at ambient $72 \pm 8^{\circ} \mathrm{F}$ or 64 to $80^{\circ} \mathrm{F}$

ii.) Calibrated Span (CS) $=$ Full Scale $=10$ VDC

B. Channel Uncertainty to MCS - General Service

i.) Relative Accuracy (RA)

Given: $\mathrm{RA}=\mathrm{RA}_{\text {avim }}= \pm 0.1 \%$ Full Range

$\mathrm{RA}= \pm 0.1 \% \mathrm{CS}$

\subsection{Modicon Analog to Digital Module (A/D)}

A. Assumptions

i.) Calibrations performed at ambient $72 \pm 8^{\circ} \mathrm{F}$ or 64 to $80^{\circ} \mathrm{F}$

ii.) $\quad$ Calibrated Span (CS) $=$ Full Scale $=10$ VDC

B. Channel Uncertainty

i.) Relative Accuracy (RA)

Given: RA $= \pm 0.05 \% \mathrm{FS}$

$\mathrm{RA}= \pm 0.05 \% \mathrm{CS}$ 
Calibration Requirements- Rosemount Temperature Channels

3.6.1.2.3 OCRWM Loop Calibration

A. Loop Tolerance (LT) Determination

$$
\begin{aligned}
\mathrm{LT} & = \pm\left[\mathrm{RA}_{\mathrm{s}}{ }^{2}+\mathrm{RA}_{\mathrm{F}}{ }^{2}+\mathrm{RA}_{\mathrm{mcs}}{ }^{2}\right]^{1 / 2} \\
& = \pm\left[0.17^{2}+0.1^{2}+0.05^{2}\right]^{1 / 2} \% \mathrm{CS}= \pm 0.2 \% \mathrm{CS} \\
& = \pm 0.2^{*} 100^{\circ} \mathrm{C} / 100= \pm 0.2^{\circ} \mathrm{C}
\end{aligned}
$$

B. OCRWM Loop Calibration Tolerance

\section{RANGE Loop Tolerance}

0 to $100{ }^{\circ} \mathrm{C} \quad \pm 0.2^{\circ} \mathrm{C}$

\subsubsection{Measurement and Test Equipment}

i.) Test Equipment Error Maximum (TEM)

1. Test Equipment1: Volt Meter

See Section 2.8.2 i., \#1

2. Test Equipment2: AMETEK 140SE JOFRA dry block

$$
\begin{aligned}
& \text { Accuracy }= \pm 0.3{ }^{\circ} \mathrm{C} \text {, Stability }= \pm 0.05{ }^{\circ} \mathrm{C} \\
& \mathrm{RA}_{\text {MTE } 1}=\text { Accuracy }+ \text { Stability }=0.3+0.05=0.35{ }^{\circ} \mathrm{C}=0.35 \% \mathrm{CS} \\
& \mathrm{RA}_{\mathrm{MTE} 1}= \pm 0.35 \% \mathrm{CS} \\
& \mathrm{RA} A_{\text {STD } 1}= \pm 0.1^{\circ} \mathrm{C}=0.1{ }^{\circ} \mathrm{C}\left(100 \% / 100{ }^{\circ} \mathrm{C}\right)=0.1 \% \mathrm{CS} \\
& \mathrm{RD} 1=0 \% \mathrm{CS} \text { (digital readout) }
\end{aligned}
$$

TEM Contribution from Temperature Standard

$\mathrm{TEM}=\left(0.35^{2}+0.1^{2}+0+0.1^{2}\right)^{1 / 2}=0.4 \% \mathrm{CS}$ 


\section{References}

\subsection{Standards}

1. ISA-S67.04, Part I, Setpoints for Nuclear Safety-Related Instrumentation, Instrument Society of America, 1994

\subsection{Vender Information}

1. Bulletin 400-10/96, 1996 - MKS Instruments, Inc.

2. letter from R. Traverso MKS Applications Engineer $1 / 26 / 98$

3. 1960501R 5/96 - Framatome Technologies

4. FTI-99-1537, letter from J. Scecina, Product Engineer, 5/5/99

5. 00813-0100-4724 Rev. AB 11/98, Rosemount Models 3144 and 3244MV Product Data Sheet

6. Fluke 743B Documenting Process Calibrator Specifications, www.fluke.com/products/, 2000 Fluke Corp.

7. Fluke 80 Series III Digital Multimeters Specifications, www.fluke.com/products/, 2000 Fluke Corp.

\subsection{HANFORD}

1. SNF-3091, Rev. 1, Cold Vacuum Drying Safety Class Instrumentation and Control System Design Description, Fluor Hanford, Inc., Richland, Washington, 1999.

2. HNF-SD-SNF-DRD-002, Rev.4, Cold Vacuum Drying Facility Design Requirements, Fluor Hanford, Inc., Richland, Washington.

3. HNF-3553, Rev 0 A, ANNEX B Cold Vacuum Drying Facility Final Safety Analysis Report, Fluor Hanford, Inc., Richland, Washington.

4. HNF-SD-SNF-RPT-007, Rev 4, Application of the Office of Civilian Radioactive Waste Management (OCRWM) Quality Assurance Requirements to the Hanford Spent Nuclear Fuel Project, Fluor Hanford, Inc., Richland, Washington, March 13, 2000. 


\section{DISTRIBUTION SHEET}

To

Distribution

Project TitleMork Order

w-4 41,

Name

R Whitehurst

BL Philipp

CS Haller

HM Chafin

JR Brehm

SNF Startup Library

CVD Library J. Jennings (2 COPIES)

CVDF Satellite Library

SNF Project Files
From

SNE-CVD

\begin{tabular}{l} 
Page 1 of 1 \\
\hline Date $7 / 25 / 00$ \\
\hline EDT No. 624970 \\
ECN No.
\end{tabular}

MSIN

$x 3-78$

$\mathrm{X} 3-78$

$\times 3-78$

$\times 3-78$

$\times 3-79$

B2-64

$\times 3-78$

$\times 3-25$

R3-11
Attach.I

Text

With All

Attach.

$x$

$\mathrm{x}$

$x$

$x$

$x$

$x$

$x$

$x$

Append $x$
EDT/ECN Only

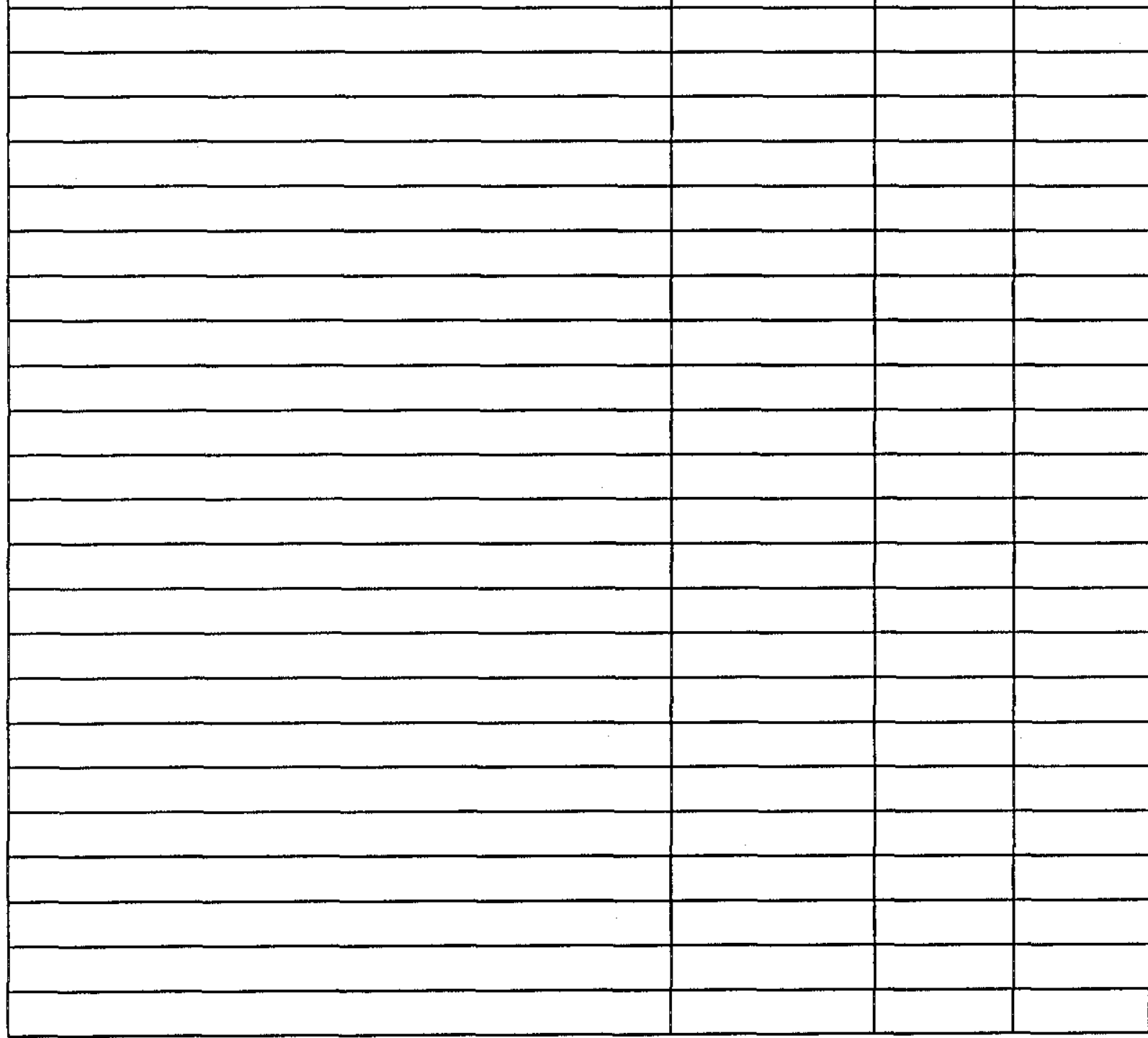

\begin{tabular}{|c|}
\hline \\
\hline
\end{tabular}

